\title{
Dialectical Models of Deliberation, Problem Solving and Decision Making
}

Douglas Walton, Alice Toniolo and Timothy J. Norman, Argumentation, to appear.

\begin{abstract}
:
Hamblin distinguished between formal and descriptive dialectic. Formal normative models of deliberation dialogue have been strongly emphasized as argumentation frameworks in computer science. But making such models of deliberation applicable to real natural language examples has reached a point where the descriptive aspect needs more interdisciplinary work. The new formal and computational models of deliberation dialogue that are being built in computer science seem to be closely related to some already existing and very well established computing technologies such as problem solving and decision making, but whether or how dialectical argumentation can be helpful to support these systems remains an open question. The aim of this paper is to examine some real examples of argumentation that seem to hover on the borderlines between deliberation, problem solving and decision making.
\end{abstract}

Keywords: Deliberation dialogue, typology of deliberation, computational models, changing the issue

\section{Introduction}

Hamblin $(1970,256)$ famously wrote that dialectical systems can be studied either normatively or descriptively. Most importantly, he stressed that the one approach is no good without the other when he wrote: "Neither approach is of any importance on its own; for descriptions of actual cases must aim to bring out formalizable features, and formal systems must aim to throw light on actual, describable phenomena." Although there are now many formal and computational argumentation systems in artificial intelligence and other fields of computer science, researchers in argumentation and informal logic have always insisted on the use of real examples as central to their pursuit. The approach of testing out their theories on real examples of argumentation from natural language texts has often been advocated by scholars in argumentation studies. Van Eemeren (2017; van Eemeren et al., 1993) has consistently insisted on the need in argumentation studies to build normative models on the basis of descriptive observations. Jacobs (1986) adopted a method of using demonstrative examples in qualitative empirical studies, as opposed to the limited value of mere illustrations. Walton (2006) consistently practiced the use of natural language examples of argumentation to build and evaluate formal argumentation models and methods.

For these reasons, it is important to recognize the value of the work needed to fit abstract dialectical systems to real examples of the argumentation they are supposed to represent formally. This paper is an exercise in that kind of interdisciplinary work. Although the normative nature of deliberation dialogue has been strongly emphasized in the recent literature, especially in computer science, the literature on deliberation has reached a point where the descriptive aspect needs more work.

This work is especially needed at the present time in connection with the new formal and computational models of deliberation dialogue that are being built in computer science and being applied to the study of argumentation. They seem to be closely related to some already existing and established computing technologies such as problem solving and decision making, but whether dialectical argumentation can be helpful to support these systems is an open question. A first step toward an answer is to examine some real examples (cases) that seem to be on the borderlines between deliberation, problem solving and decision making. That is the aim of this paper.

By presenting a series of realistic examples of deliberation, in this paper, we also seek to form a typology of deliberation dialogues. The observations from our examples are used to suggest that argumentation researchers and philosophers have been thinking about deliberation in simplistic ways. We argue that to better understand and contextualise 
argumentation components that make up realistic deliberations, it is necessary to distinguish between different kinds of deliberations. The objective of our typology is to provide a characterisation and a frame of reference for different types of computational deliberation dialogues, with scope for analysis and extension of existing frameworks to be able to handle a more comprehensive set of problems.

The typology we propose is informed by the study of realistic examples and dialectical argumentation theories, focussing on how different types of dialogue, in particular persuasion, have influenced deliberation dialogue models, and how this reflects on computational models. We focus on deliberation as a whole process including how the problem is set out, how and when options are constructed, selected, and advanced in the dialogue. We build on models of deliberation dialogue in artificial intelligence, inspired by the McBurney, Hitchcock and Parsons (MHP) model (McBurney et al., 2007), and other computational argumentation-based deliberation systems (e.g. Kok et al., 2011) including a model that recently arose from this literature (Walton, Toniolo and Norman, 2016). Many deliberation models have the aim of making a choice between a set of alternatives fixed at the outset, but here we explore others that aim at how to do something that requires action because a problem has arisen. In this paper, we approach problem solving deliberation on how to do something, and contrast it with the deliberation of the dilemma kind often emphasized in philosophy. When participants are faced with solving a problem, dialogue is important to enable participants to plan and act in collaboration to solve the problem. We hold that this form of dialogue is a type of deliberation and is a fundamental part of the problem solving procedure.

Furthermore, our typology is closely linked to features that characterize established computational research in problem solving and decision theories, in which differences and similarities influence the type and purpose of the options advanced in the dialogue, and shape different dialogue models. A similar link between decision theories and deliberation has been previously explored in Van der Weide's thesis $(2011)$. Van der Weide $(2011,110)$ uses argumentation schemes, such as the scheme for practical reasoning, and a dialogue framework for supporting decisions. On this approach, deliberation is defined in a different way than in the dialectical theory of Walton and Krabbe (1995), where deliberation is modelled as a type of dialogue in which a group of agents is arguing to try to decide what is the best course of action in a situation requiring a decision to be made (Walton, Toniolo and Norman, 2016). However, van der Weide $(2011,110)$ defines deliberation as practical reasoning to identify what goals an agent should try to achieve. This way of proceeding defines deliberation as one special part of a rational agent's decision making, and therefore does not seem to be a dialectical approach. However, van der Weide $(2011,137)$ also offers a dialogue framework for supporting decisions, stressing that human decision makers often use argumentation to resolve conflicts and to justify a choice to others as well as to themselves. But in this system, agents arguing about the consequences of actions they are thinking of undertaking require an argumentation system that works at a meta-level of deliberation. The main level of argumentation takes place in what is called the decision-support dialogue that helps the user judge what is the best course of action according to the system. In this framework, the system and the user collaborate on finding the best decision.

What does 'deliberation' mean then, in contrast to the way this term is used in the formal argumentation model of Walton and Krabbe and subsequent formal models of deliberation dialogue such as the MHP system? Decision-support systems and deliberation dialogues have similarities but also differences. But van der Weide $(2011,139)$ cites the MHP model with approval, conceding that a mixture of these technologies could improve deliberation when working with realistic natural language examples of the argumentation in which people typically engage. This was already indicated by the authors of the MHP model, 
who recognized that there is a good deal of shifting from information seeking dialogue to deliberation, or from deliberation to persuasion dialogue.

On the other hand, van der Weide uses the term 'deliberation' in a different way from the way it is used either in the MHP model or in subsequent ones such as the Walton, Toniolo and Norman (2016) model. According to van der Weide (2011, 139), the decision-support dialogue should be classified as an inquiry because the system and the user collaborate on finding the answer to the question about what decision to make, but it should also be classified as a deliberation type of dialogue because it determines what decision best fulfills the goal of the agent. This dual meaning of 'deliberation' is hard to reconcile with the one given by Walton and Krabbe (1995) unless shifts from inquiry to deliberation are taken into account.

The differentiation between the ways these formal systems use the term 'deliberation' and attempt to distinguish between inquiry or deliberation and decision making poses a problem for applying formal argumentation models to real examples of argumentation. Does deliberation represent a formal dialectical structure in which argumentation takes place alongside the other types of dialogue, or does argumentation merely take place in decision making when pro and con arguments and evidence concerning a choice of actions needs to be weighed and taken into account? The best way to approach this problem is to analyze the argumentation in some real (not too simple, but not too complex) examples of natural language deliberative dialogues to see how they ought to be classified. This job is not an easy one because it requires fitting the abstract properties of formal models of dialogue to realistic examples (cases) of natural language argumentation, but its importance has been recognized by leading argumentation theory theorists (Van Eemeren, 2017).

Our analysis of real examples and formal models of dialogue results in a set of characteristics that form a typology of deliberative dialogue, in particular distinguishing between problem solving deliberation and decision making deliberation. The differentiation between types of deliberation represents a context for the initial findings presented by Walton and Toniolo (2016). The work presented in this paper extends this previous research by providing grounds for formulating a typology of deliberation dialogue. Here we go further, and through the use of examples, we provide a more comprehensive view of the features that make up different types of deliberation. We suggest that further characteristics must be modelled in computational frameworks such as how to revise the opening issue and how the introduction of new information influences the identification and choice of new actions. In our previous research (Walton et al, 2014; Walton, Toniolo and Norman, 2016), we introduced the idea of extending the McBurney, Hitchcock and Parsons model, to include the possibility of considering an open knowledge base, that allows participants to record the changes of circumstances during deliberation. In this paper, we reflect in particular on the need to adapt and expand computational models to represent the problem solving type of deliberation.

The remainder of this paper is organised as follows. We review related literature in persuasion and deliberation in Section 2, to provide an initial characterisation of different deliberation forms, showing aspects in which persuasion has influenced deliberation. In Section 3, we present and examine six examples of realistic deliberation dialogue to demonstrate the need for a typology of deliberation. Section 4 provides a reflection on the examples proposed, leading to our proposed typology being further substantiated by existing literature. In Section 5 we reflect on existing features of computational models of deliberation, proposing extensions that we envisage useful for a more comprehensive model. Section 6 presents a summary of our dialogue typology and our final remarks.

\section{Persuasion and Deliberation}


In the standard models of persuasion dialogue currently accepted in computational argumentation, there are three stages: an opening stage, an argumentation stage, and a closing stage (Prakken, 2005). The so-called issue or conflict of opinions is set in place at the opening stage, and then the arguments on both sides go back and forth during the argumentation stage. At the closing stage, it is decided which side had the strongest supporting arguments for or against the issue. The same model applies in legal argumentation in a trial. The so-called burden of persuasion is set at the opening stage by law, and then during the argumentation stage as both sides put forward their arguments, there is an evidential burden that can shift from one side to the other. The outcome is decided at the closing stage depending on which side is taken by the judge to have met its burden of persuasion (Prakken and Sartor, 2009).

In the ancient manuals on rhetoric, this approach was called the stasis theory (Hohmann, 1989). The so-called stasis (or status, as it is called in Latin) is the issue set in place at the opening stage of a speech, and the rest of the speech is confined to pro and con arguments that are relevant to showing whether this designated proposition is true or false. Setting the status in place at the opening stage has normative implications that apply during the argumentation stage. Suppose it can be shown that one of the arguers has wandered away from the topic and moved onto some other distracting or emotionally exciting matter that is irrelevant to proving whether the status proposition at issue is true or false. In such a case, a valid normative criticism would be that this line of argument is irrelevant. Relevance, just as in law, can be described as a failure of an argument to carry sufficient probative weight to either prove or disprove the proposition at issue, as determined by the status (Walton and Macagno, 2016).

In the standard models of deliberation dialogue accepted widely in the computational argumentation literature (Reed, 1998; McBurney et al., 2007; McBurney and Parsons, 2002), deliberation is different from persuasion dialogue but also similar to it in some respects. In deliberation, the issue is one that confronts an agent in a particular set of circumstances where choice is required. A typical deliberation of this sort often emphasized in the philosophy literature concerns a dilemma, a two-place issue, such as the decision whether physicianassisted suicide should be legally permitted or not in a jurisdiction. Further differences include the absence of fixed initial commitments of participants, and the collaborative nature of participants seeking a solution that is optimal for the group rather than for the individual (McBurney et al., 2007). Also, there are different pre and post conditions for speech acts in the two types of dialogue (Atkinson et al., 2013). However, computational models of deliberation have often been adapted from persuasion models to deliberation and inherit some of their characteristics. An example of this approach can be found in the work of Kok et al. (2011) which extends the model of Prakken (2005).

One similarity between the standard approach to deliberation dialogue and persuasion dialogue is that both types of dialogue have three stages (McBurney and Parsons, 2002), where the issue is set at the opening stage, stays in place during the argumentation stage, and is used to determine at the closing stage which side was victorious. The sharing of the fixed status property, however, seems to become open to questioning on some models of deliberation. By examining different examples, in this paper, we show that the status property may be subject to changes during the deliberation and we argue that models of deliberation should account for such a revision. The fact is that in a deliberation the question (where should we eat?) remains fixed, but new answers may be introduced during the dialogue, and that is an important characteristic of deliberation, which does contrast with persuasion (let's eat at Gino's) where the answers are fixed. 
On the formal argumentation model of deliberation proposed by Walton, Toniolo and Norman (2016), a strong emphasis is placed on the importance in a rational deliberation procedure of the participants taking the changing circumstances of the case into account. The argument for moving to this theory is that it should be a central characteristic of any normative model of deliberation that the procedure should be flexible during the argumentation stage. Plans that may have previously been set in place can then be quickly modified to adapt to the necessities of new circumstances subject to rapid changes.

An implication of this approach is that the choices that are set in place at the initial stage of deliberation may be changing as the dialogue moves forward and participants become aware of new or revised circumstances. Suppose that an agent or a group of agents is making a decision in a typical dilemma case where there is only a choice between two options. Later on, suppose that new information comes in because of observations of changing circumstances. At this point, there is no possibility to account for how to deal with the situation in which this new information indicates that there is a third option that was not present before. For example, suppose that Jack and Jane are at the ice-cream shop and have to make a decision on what kind of gelato to buy. In the shop, however, all the containers of gelato displayed are empty except for one containing chocolate gelato and another containing vanilla gelato. Jack asks Jane about what flavor of gelato she is going to buy, adding that she is a good judge of gelato and he is not sure on what flavour he prefers among these two. Just as they are about to decide, the owner of the shop brings in a new container of gelato containing cherry gelato, opens it, and puts it in the display case alongside the other two containers. With these changing circumstances being observed by the agents making the choice, a third option becomes available to them. The status that was set in place in the first instance has changed, so that now a choice needs to be made between three options. This may also happen the other way around, for example in a case where there were originally three types of gelato available, but one was emptied by previous consumers while the agents are making the decision, reducing the choice to a decision between two flavors.

This case is a very simple example for illustrative purposes, but it would seem that the same principle applies to much more complex deliberations, where for example different participants exchange information of individual intentions to perform certain actions that were not previously known by the other participants. Another example is a decision about investments in a stock market, where significant changes are made rapidly in the choice to be made. It is problematic to deal with realistic deliberations in which agents may be asked to make a decision between two options but then an investigation of the circumstances of the case reveals other relevant options that might even be better than the original ones. There is even a traditional informal fallacy associated with this kind of problem called the black-andwhite fallacy, the fallacy of reducing a decision where there should be a range of options to a simplistic yes-no formulation of the question to be decided (Walton, 1991).

Furthermore, some complex forms of deliberation dialogue may proceed by highlighting that the goal cannot be achieved in the present circumstances. Participants would then instead focus on subgoals, in the form of sub-issues, and attempt to identify actions that can be directly carried out and that will ultimately lead to an action that can carry out the primitive task. Once these subgoals are attained by further actions there will be a route opened up to the carrying out of primitive tasks that can achieve the original goal that was formulated at the beginning.

These considerations suggest that there is a key difference between persuasion and deliberation dialogue that has not been previously noticed. In persuasion dialogue, the original status set in place at the opening stage remains fixed through the argumentation stage in order to make the arguers stay on point. In deliberation dialogue however, because of the vital need for flexibility, a reformulation of the issue needs to be made that is variable during 
the argumentation stage so that options can be added to it, or deleted from it, as relevant circumstances change. This suggests that relevance needs to be defined in a different way in deliberation dialogue from the widely accepted way it has been viewed in persuasion dialogue.

We have so far set the basis for at least three types of deliberation: a) a "dilemmalike" deliberation dialogue, similar to the dilemma problem, where the stasis principle applies and the dialogue moves between pros and cons to decide whether to do something or not; $b$ ) a "static decision making" deliberation dialogue, where a fixed set of actions are moved for discussion at the opening stage but remain fixed during the dialogue aimed at making a decision on what to do; and $c$ ) a more flexible approach, a "dynamic" model of deliberation dialogue in which the status is subject to changes during the dialogue in both alternatives and sub-issues, and in turn the new information acquired during the dialogue guides what actions compose the new status. In the next section, we consider this last type of dialogue more closely and drawing from real examples, we attempt to define different classes in which deliberation is a dynamic flexible process of coming to a conclusion on what to do. Before presenting our example and analysis, however, we characterise the type of arguments that lie at the basis of all different types of deliberation introduced.

In this paper, we focus on a particular argumentation scheme that is fundamentally important, and that is the scheme for practical reasoning. Practical reasoning is sometimes a purely instrumental form of argumentation in which the circumstances pose some sort of practical problem, such as fixing a printer, and means for solving the problem. Different approaches for practical reasoning have been proposed in the literature for example by Hulstijn and van der Torre (2004), Rahwan and Amgoud (2007), and Toni (2008) presenting general forms of such instrumental arguments. In many instances, when values are involved as well as factual matters, the value-based scheme of practical reasoning is useful to represent a more structured form of practical reasoning (Atkinson et al, 2006; Bench-Capon, 2003; Atkinson and Bench-Capon, 2007). The values are often used as means to evaluate options, and may be used in a form of persuasion dialogue over actions (Atkinson et al., 2013). On the other hand, values can be ignored if the case is one that requires only considering how to find a means to fulfill a goal without considering the values of the agents. An adapted version of the argumentation scheme for value-based practical reasoning as proposed by Gordon et al. (2018) is presented below.

VALUE-BASED PRACTICAL REASONING

Premise 1: I have a goal G.

Premise 2: $G$ is supported by my set of values, $V$.

Premise 3: Bringing about $A$ is necessary (or sufficient) for me to bring about $G$.

Conclusion: Therefore, I should (practically ought to) bring about A.

Critical Questions:

CQ1: What other goals do I have that might conflict with G?

CQ2: How well is $G$ supported by (or at least consistent with) my values V?

CQ3: What alternative actions to my bringing about A that would also bring about $G$ should be considered?

CQ4: Among bringing about $A$ and these alternative actions, which is arguably the best of the whole set, in light of considerations of efficiency in bringing about $\mathrm{G}$ ?

CQ5: Among bringing about $A$ and these alternative actions, which is arguably the best of the whole set, in light of my values $V$ ?

CQ6: What grounds are there for arguing that it is practically possible for me to bring about $A$ ? 
CQ7: What consequences of my bringing about $A$ that might have even greater negative value than the positive value of $G$ should be taken into account?

Generally, the argumentation scheme for practical reasoning is able to represent the posing of the initial issue as a goal that can be achieved by alternative actions. In existing computational work, such as Black and Atkinson (2009), this scheme is instantiated during the dialogue as means to propose different alternative solutions to make a decision on the best course of action to adopt. In the examples presented in this paper, this scheme can be used to model arguments that underpin all different types of deliberation dialogue proposed.

\section{Realistic dynamic deliberation}

In this section, we provide a set of examples that show differences in the kind of deliberation dialogue that participants can take part in. This forms the grounds for a more comprehensive typology of deliberation providing scope to contextualize existing argumentation-based deliberation and consider whether further refinements and additional characteristics are needed to better represent more realistic deliberations.

In the analysis, we use two graph representations, in different or combined formats. The first type of representation is the argumentation graph, used to represent the structure of the argumentation These argument graphs are a form of argument mapping central to informal logic and argumentation theory and are useful in our effort to better comprehend realistic deliberations. The MIT Collaboratorium ran an example on the topic of the future of biofuels in Italy which was evaluated by a test run performed in December 2007 at the University of Naples with a community of 220 graduate students (Klein and Iandoli, 2008). This experiment suggested that recent argumentation tools, such as argument mapping, can help make complex deliberations reach higher levels of quality.

The argumentation graphs used in this paper follow the conventions of the Carneades Argumentation System (Gordon, 2010). The rectangular nodes in a Carneades argument map represent propositions (premises and conclusions) while the round nodes represent arguments to or from propositions (see Fig.1a). Relationships between arguments appear in circular nodes, and they are numbered, such as a1, a2, and so forth. A pro argument has a plus sign, such as $+\mathrm{a} 1$, and a con argument has a minus sign, such as -a2. This form of representation is similar to the format used by the latest version 4 (http://carneades.github.io/) of the Carneades Argumentation System. The most significant feature for the purpose of this paper is the use of the six-sided nodes to represent issues that can change over a sequence of argumentation.

The second type of graph represents the dialogue process as a workflow, from the starting point (ellipses), to the end points (octagons), where different steps are represented as yellow (grey in print) rounded rectangles (see Fig. 1b).

\subsection{The example of buying a car}

A common example that a theory of deliberation should be able to handle is a decision making on which car to buy. The example presented below is derived originally from Brewka and Gordon (1994) and later variants can be found, for example in Gordon (2015).

Suppose Mary and Bob have decided that their car which they have had for ten years has become too costly to maintain and requires a complete overhaul, and so they decide that it would be better to buy a new car. Mary prefers the Volvo while Bob prefer the Porsche. They discuss which would be the better choice. She argues that the Volvo is safer. Bob argues that 
the Porsche is more sporty. This discussion goes on for a while considering pros and cons of these two cars with seven argument being presented. But then they discover the car buying guide of Consumer Reports, which offers some relevant information about frequency of repair records and the outcomes of crash testing.

Bob and Mary share several values that are important for making their decision: safety, costs and style. If we were to put these values in a priority ordering, safety would be at the top for Mary, while style would be at the top for Bob. But both think that cost is also important. In some AI argumentation systems, weights are attached to these values for each party (for example, in Carneades as discussed by Gordon (2018)), but here we keep the example simple for purposes of illustration. There is also another argument to be considered, $\mathrm{a} 4$, that has come forward because both Bob and Mary have seen numerous Volvo ads claiming that the Volvo is a very safe car. This provides an argument that supports Mary's claim that the Volvo is safer.

When the deliberation began, at the opening stage, as shown in Figure 1a, the issue for Bob and Mary is whether they should buy a Volvo or a Porsche. The nodes labeled a1, a2 and a4 are shown as pro arguments, as indicated by the plus sign. But another argument a3 is also presented. Both know that the Porsche is more costly, and this constitutes a con argument against the Porsche for both of them, because they share the value of saving costs.

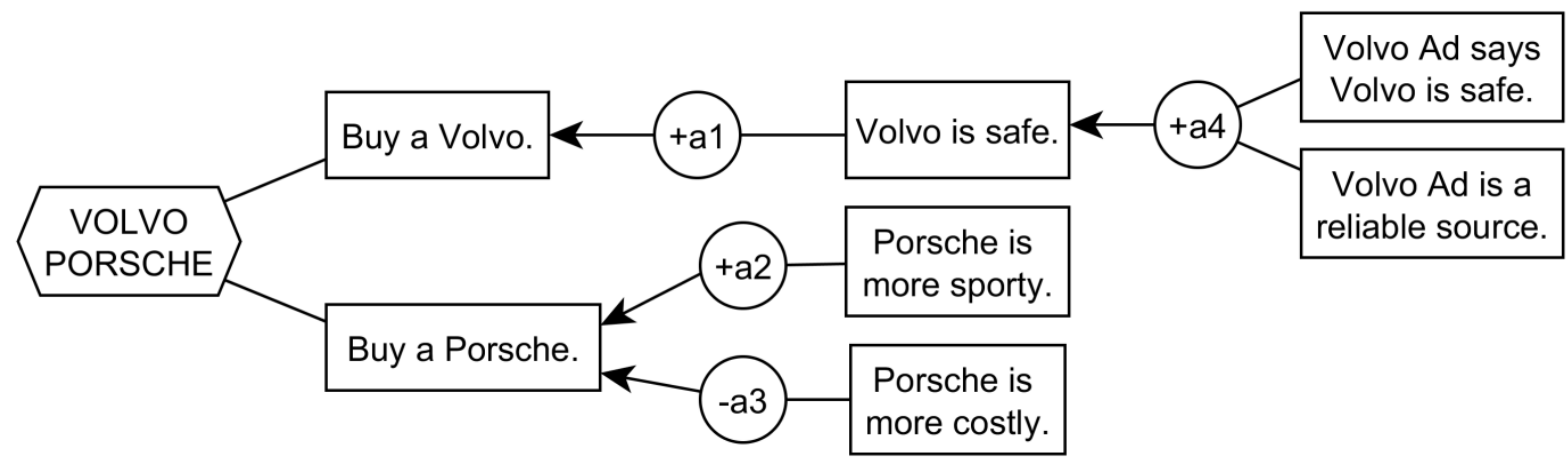

Figure 1a: Initial Issue and Argument Map in the Car Buying Example

It is at this point where the new information comes in from the Consumer Reports car buying guide. They find in the buying guide that the well-known Toyota Camry has the best frequency of repair record, and since both know from experience that repairs to a car can be costly, new information has come into the dialogue that supports the decision for buying the Camry. Mary and Bob also find out from reading the car buying guide that the Camry scored high in the latest crash tests. This information is also relevant to their deliberation because it suggests that the Camry is very safe.

Clearly Mary and Bob have to take this new information into account. At a particular point in the deliberation they have collected some facts that are relevant to their decision, and these facts, along with the discussions they have about them, reconfigure the deliberation. It is no longer simply an issue between buying a Volvo or buying a Porsche. It is now an issue about whether they should buy a Volvo, a Porsche or a Camry, as shown in the three option choice shown at the left of Figure 1b. Not only has new information come in, but this information can be linked together into arguments that support or attack the three options set into place, as shown in the argument diagram of Figure 1b. The new information has resulted 
in an improvement of the argumentation in the deliberation dialogue. We note also that not all car types that score high in safety and maintenance are brought into the dialogue, but a single additional one. If the dialogue were to continue by indetifying that the Camry also was not an adequate choice, Mary and Bob could have introduced a further additional alternative by revising the issue again.

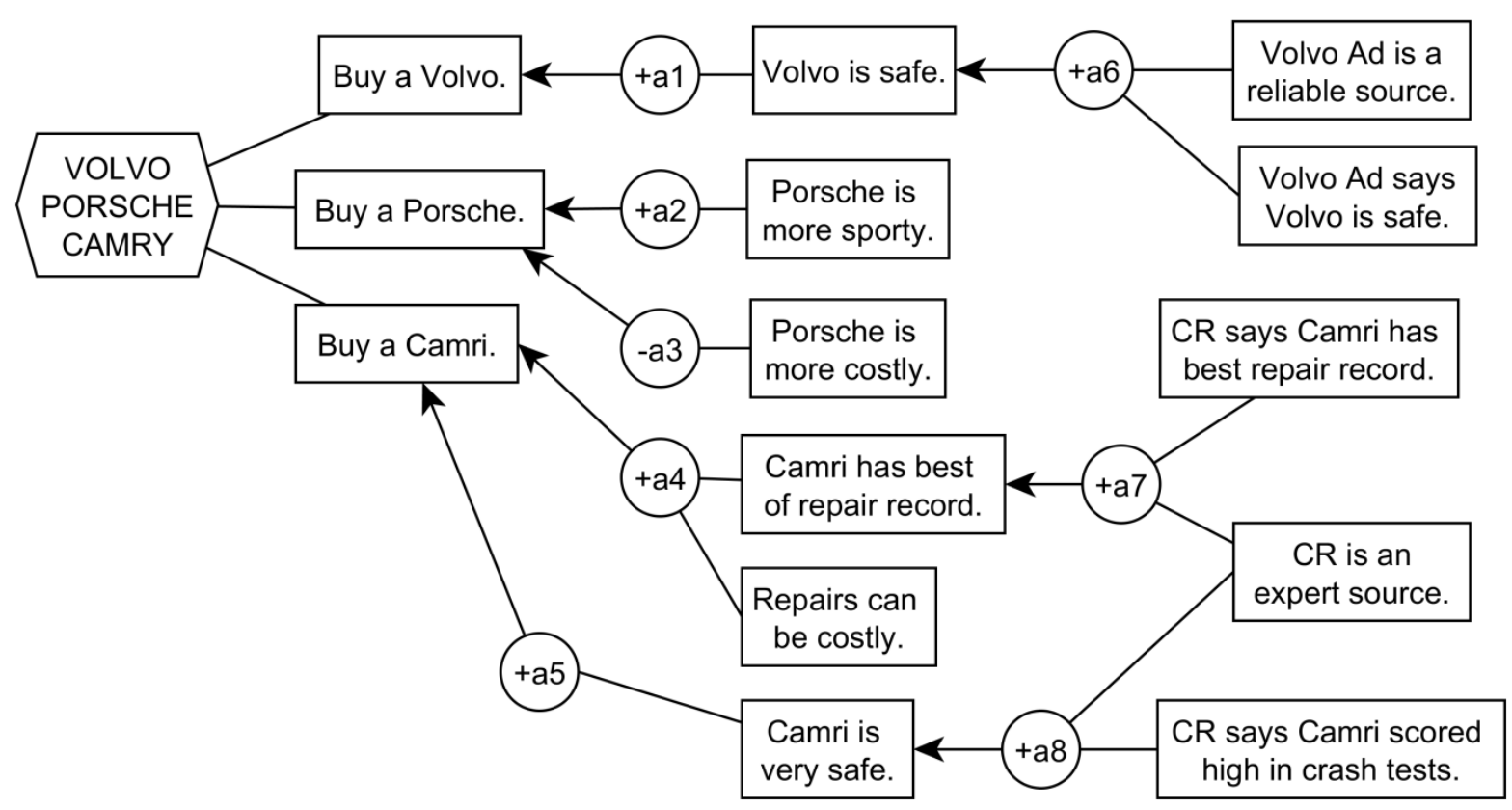

Figure 1b: Revised Issue and Argument Map in the Car Buying Example

This deliberation started out as a choice between two cars, where each of those options can be represented as an instance of the practical reasoning scheme, followed by a sequence of argumentation in which the pros and cons of each option are considered. But then, as more information came in about the situation, the original decision to be made was reconfigured. At this point it was seen that the options needed to be re-evaluated. The revision of the issue informed by the information and discussion shared during the dialogue led to the identification of new relevant alternatives for restructuring the deliberation. This should be seen as an evidential deepening of the deliberation dialogue. The dialogue process is shown in Figure 1c.

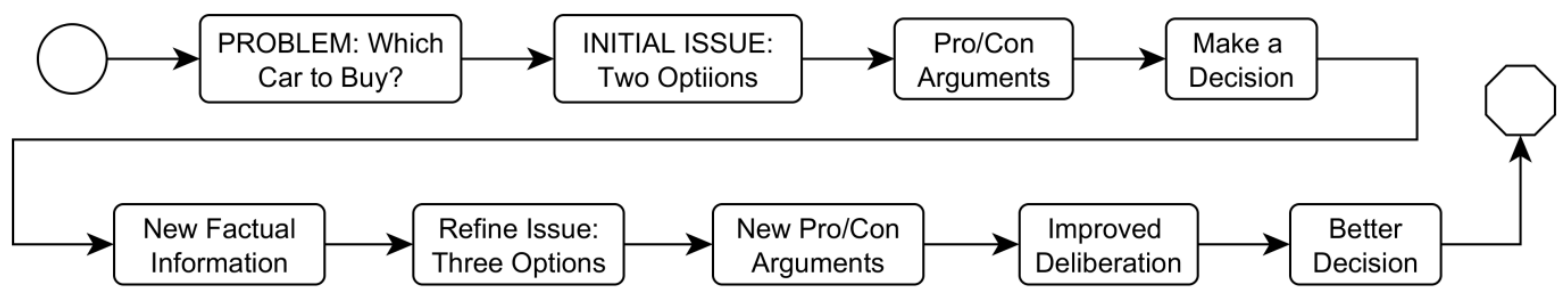

Figure 1c: Dialogue Process in the Car Buying Example

There are formal and computational argumentation systems that allow for the refinement of the initial issue in a deliberation to enable the participants to reformulate the issue (Atkinson et al., 2013). For example, version 4 (http://carneades.github.io/) of the Carneades Argumentation System has this capability, from which we draw to represent our argument 
diagrams. During a deliberation dialogue, new information can come in, and once that happens, new alternatives may be formulated, and new pro and con arguments may be produced that are relevant in the deliberation dialogue. As new information comes in, and new arguments are produced based on that information, new speech acts are performed that are regulated by the procedural rules of the deliberation dialogue. A more generalised overview of this process is presented in Figure 1d.

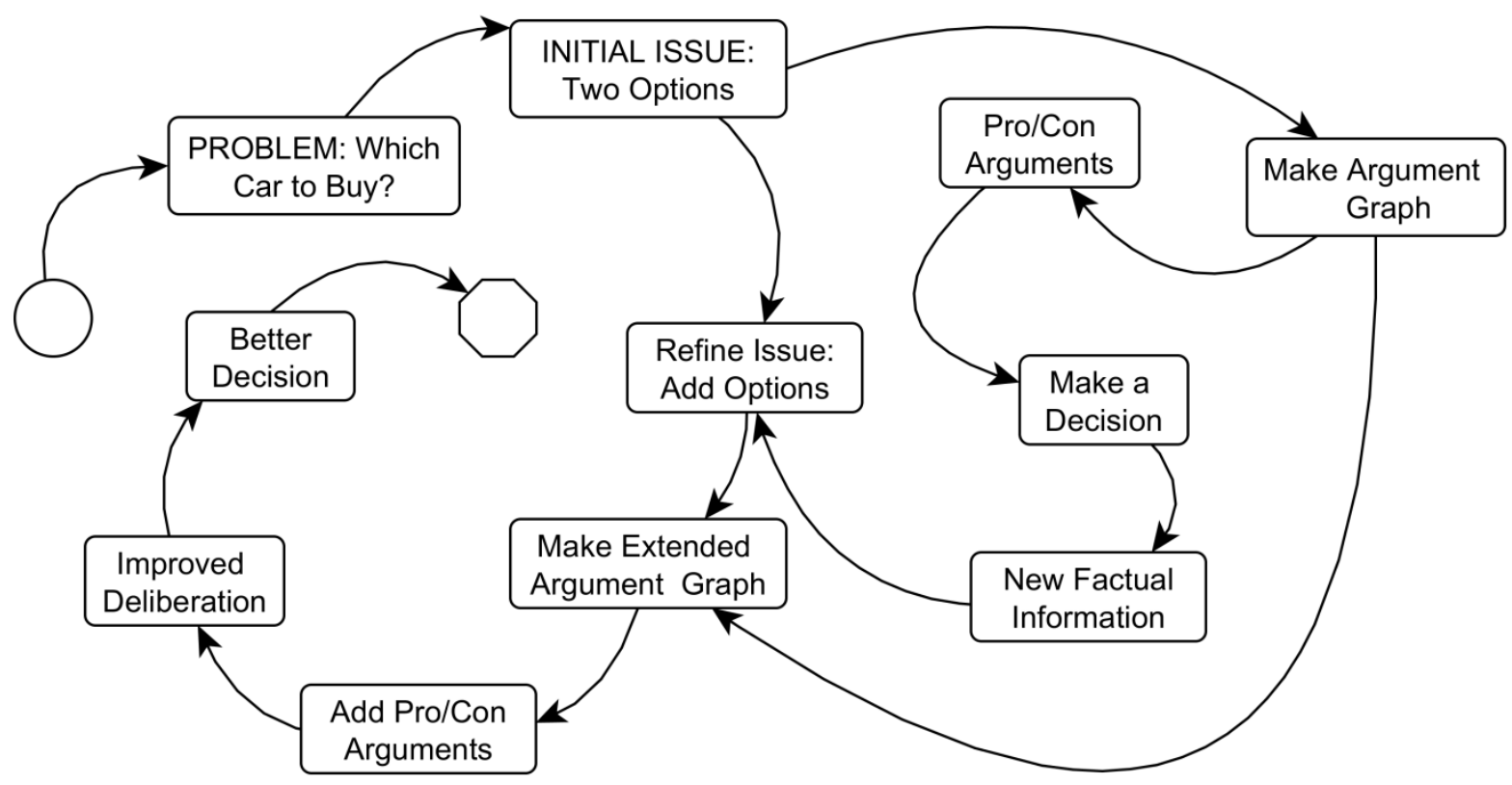

Figure 1d: Generalised Dialogue Process in the Car Buying Example

\subsection{The printer example}

In this case, Brian had a problem with his printer (Walton, Toniolo and Norman, 2016, 160161; Walton, 2015, 149-153). Whenever he scanned a document using the automatic document feeder, a black line appeared down the middle of the page. Brian considered sending it back to the manufacturer, but that might mean remaining a long time with no printer. Brian decided to try to fix it himself. Following the instructions in the troubleshooting guide, he opened the scanner cover and located a small strip of glass with a plastic cover at the left of the glass. He peeled the plastic cover off and found a small black mark in the middle of it. Using a soft cleaning pad, after asking his wife about it, and getting help from her, Brian managed to remove the black mark. He reapplied the plastic cover to the strip of glass and tried to scan a document. He then found that there was no black line down the middle of the page.

Deliberation is about solving a problem. Deliberation is something that requires action because a problem has arisen. In the printer example, the scanner is not working properly, and cannot be used in its present state because of the black line down the middle of the page. So something has to be done. The deliberation in this situation is between the option of trying to fix it and sending it back to the manufacturer. This is also individual deliberation that can be seen as a process in which one thinks aloud by asking questions and then answers them himself. For this reason, we take this example as capable of being modeled as a deliberation 
dialogue, even though critics tend to insist that it is only an instance of solitary reasoning that cannot fit any formal argumentation dialogue model.

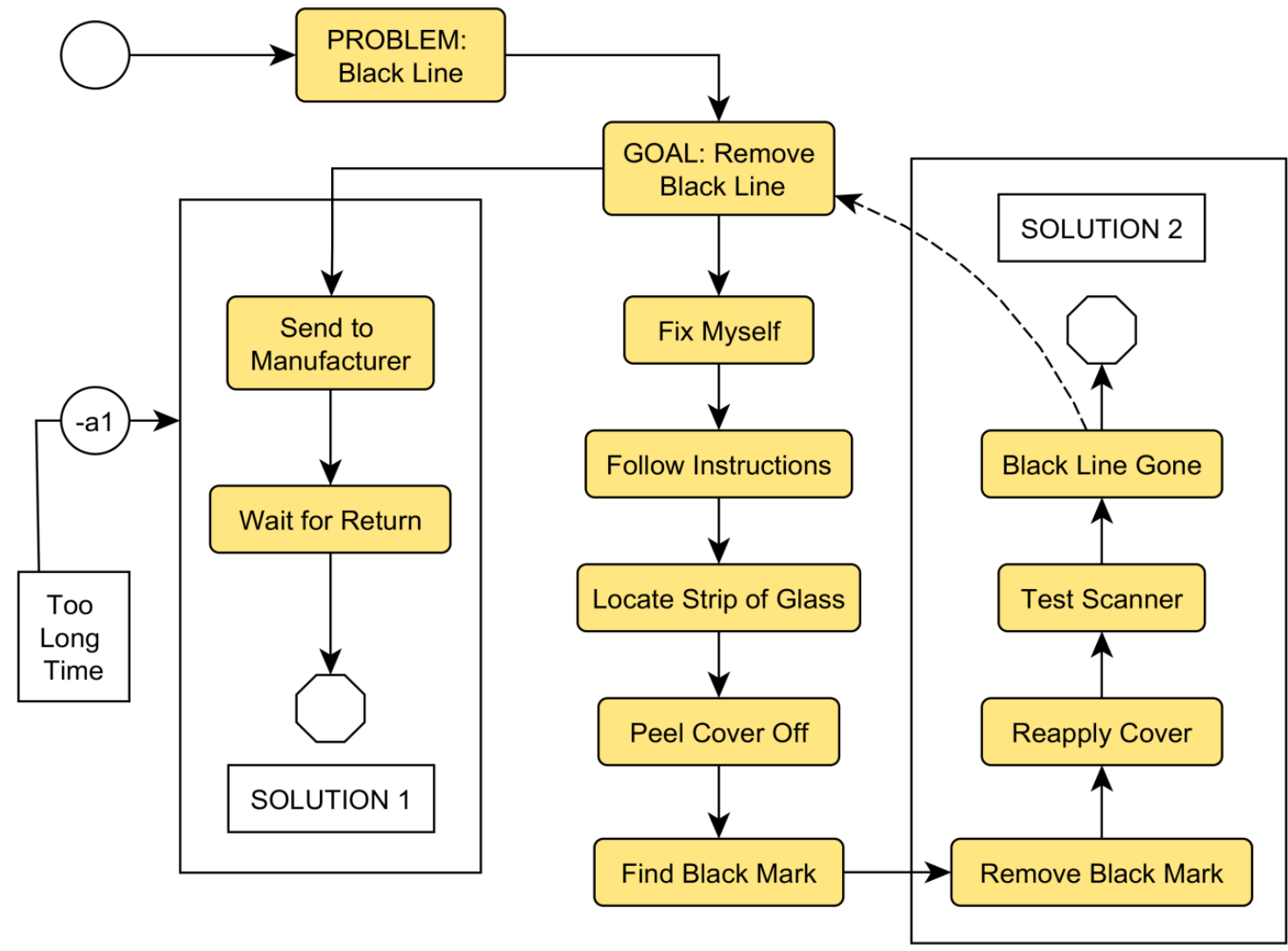

Figure 2: Problem Solving in the Printer Example (Dialogue Process and Argument Graph)

On our way of modeling it, the broken printer example represents a deliberation by posing a problem that needs to be solved by articulating and comparing pro and con arguments, as represented in the process shown in Figure 2. With the broken printer example, we have shown an example of dynamic deliberation that is used for problem solving. We refer to this type as Problem Solving Deliberation: a deliberation dialogue aimed at solving a problem in which the issue is continuously revised due to new information and solutions identified during the course of the dialogue.

In this case, we can use the scheme for practical reasoning for the problem solving kind of deliberation where the goal of removing the black line can be achieved by either of the two solutions. The initial issue in the dialogue is posed as a goal. Each solution can be posed as an instantiated action or sequence of actions, as means to achieve the goal. The critical questions CQ1 and CQ2, that question whether there are other goals or whether the goal is worthy to be pursued, may be used to enter a phase of revision of the initial issue. For example, if Solution 2 was not leading to a positive outcome, Brian may decide that there is no point in fixing the printer and change the goal to buying a new printer. However, while the practical reasoning scheme is largely used in the computational community, the difference highlighted by this example lies in when this scheme is instantiated. Here we could have a scheme representing Solution 1 (on the left-hand side of Figure 2), and only after that is refused, Brian elaborates Solution 2 (on the right-hand side of Figure 2). This contrasts with, for example, the use of this practical reasoning scheme in dialogue models such as that of Black and Atkinson (2009) in which it is assumed that Solution 1 and Solution 2 are known by the agents from the beginning. Although in the printer example we may also accept that 
both options will be elaborated from the initial stage, there are other examples, such as our next case study, in which an alternative cannot be elaborated in advance as the circumstances for finding alternative solutions are not known at the outset. In such cases, the new circumstances would require a revision of the set of actions available and consequently of the underlying attack and support relationships between arguments in order to represent the realistic dialogue.

\subsection{The disaster example}

Two agents $x$ and $y$, representing a local authority and a humanitarian organization respectively, are concerned with the repair of the water supply in a location that has suffered catastrophic damage. Agent x proposes to stop the water supply to the location. Agent $y$ argues that there is a need for water in that location to run a field hospital, which is required to aid disaster victims. Agent x proposes that the supply of water to the location must be stopped because it is not safe since the water may be contaminated. Furthermore, $x$ raises safety concerns when discovering that the water supply is used for running a field hospital in the location due to the intention of using excavators in the same location at that time. To solve the problem, $x$ and $y$ need to modify their individual plans, constrained by their goals, the known circumstances of the case, and values such as public safety.

The example summarized above is part of a larger example (Toniolo, 2013). The example clearly involves practical reasoning because each organization has its goals and is compelled to act in accord with these goals given the circumstances and the means available. Several solutions to address conflicts are discussed by Toniolo (2013). For example, the field hospital might be set up in a different location where there is no danger of water contamination. One of the water supply sources might only be used when arrangements can be made for the excavators not to be in use during periods when water is available. Each side needs to build its plan in consultation with planning of the other side. Conflicts of this sort need to be identified for good planning and to be resolved or dealt with by arguments put forward by each side in collective deliberations.

In Figure 3, we present a diagram of the dialogue process above and its corresponding argument diagram below. In this figure, the rectangles contain propositions while the circles contain arguments joining some propositions to others by inferences from premises to conclusions.

Within the larger example, Figure 3 represents an instance of dialogue involving two incompatible courses of action, something has to be done one way or the other, and the issue concerns public safety. Moreover, each side has arguments to back up their position. In particular, the deliberation involves a decision between building a water line to the field hospital site or not. But the problem, when expressed this way, may be misrepresented as simply one of putting the water line there or not. It is really a problem of how to deal with the conflict between the needs of the two agents. Agents engage in deliberative dialogue and attempt to solve conflicts by modifying individual plans to accommodate other agents' constraints. The information shared about individual plans is fundamental to derive the identification of alternative courses of actions, and as for the printer example, alternatives are formed after a solution or a part of it is considered unsatisfactory to solve the problem. These new alternatives are still considered relevant as the deliberation proceeds, as they are formed in light of the discovery of conflicts or changes in the circumstances and they contribute to the issue of how to solve the problem rather than to the decision of whether or not the agents should perform an action. 
In this example, we note an additional approach to revising the issue, where new information, such as the need for a field hospital, opens a sub-issue which was not declared at the start but becomes relevant as the problem solving process unfolds. Through new information we identify that there is a sub-issue to be solved, i.e. where to build the hospital, which in turn could open up many new alternatives that need to be considered. In a deliberation dialogue not all the subgoals are declared at the start. These subgoals are elicited as steps needed to move forward to find a direction to lead to a solution to the original goal. Although this could lead to a separate dialogue, it commonly takes place within the original deliberation dialogue as a means to solve the original objective. For example here, if agent $y$ agrees to build the field hospital in a different location, the other agent $x$ will be able to stop the water supply and avoid contamination.

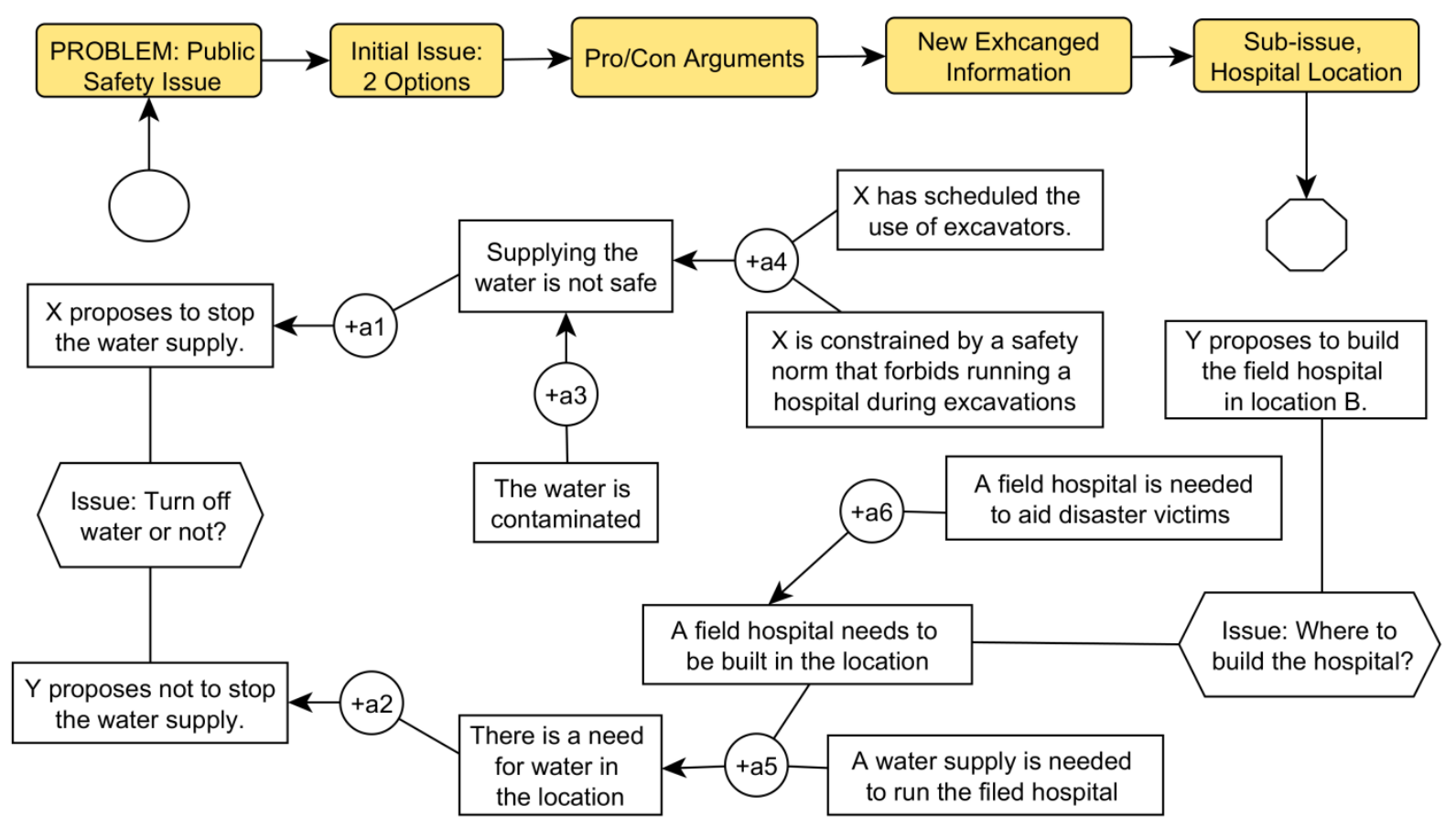

Figure 3: Problem Solving in the Disaster Example

\subsection{The example of collaborative design and construction}

An interesting example of deliberative dialogue is that of collaborative design. This topic is relevant in our paper as the focus of the dialogue is to solve a problem, where different designers have to collaboratively create a new object by considering some initial requirements. There are numerous studies of early stage collaborative design activities according to the different subjects of design, which may range from engineering, the built environment, informatics and so on. Generally, these studies share the common objective of understanding the cognitive processes of designers while they explore alternatives for new products. These types of studies involve initial observation of individuals in teams, discourse analysis through transcripts and video recordings, and definition of a protocol that may be employed to support these activities more effectively. As discussed by Stumpf and McDonnell (2002), the interaction between teams in early design activities involves an argumentation process. Aakhus and Jackson (2005) study the design process of a device as a paradigm to inform a theory for design to support communication and interaction. In this paper, we are also interested in the type of dialogue that occurs between designers. The phase 
of design that focusses on the identification of the product is a deliberative dialogue underpinned by practical reasoning as it involves the decision of what to do to realise the idea (Leon and Toniolo, 2015). The dialogue presents a record of the process of design, therefore it represents an example of how participants move forward to solve a problem. In particular, these dialogues have no initial set of options: alternatives are formed and discussed, accepted, refuted or reconsidered while the dialogue takes place.

Here we present an interpretation of a dialogue in which two designers, Mark and Jane, aim to design a litter-disposal system for the passenger compartment in a railway train. The objective of the system is to be both convenient for the passengers to deposit garbage and easy for the cleaners to collect the garbage. The text below is extended from a transcript of the design process from a collection of tasks recorded by Yao (2007); while the original is the transcript of a monologue from an individual designer thinking aloud, here we assume that two designers are reinterpreting such a dialogue to better illustrate the deliberation process.

Mark: Where should we put the bin? We can put it under the table.

Jane: But it will affect the movement of the passengers' legs.

Mark: We need to consider that it needs to be easy for the cleaners to pick up the rubbish. And cleaners walk along the corridor.

Jane: We could put it under the seats.

Mark: Under the seat is not convenient for being picked up as the passenger would have to move away, we could put it along the aisle beside the chairs.

--- Drawing of bins beside chairs

Jane: Is beside the chairs a good place? How would the bin collect the rubbish?

We can have a bag or just a bucket to be emptied.

Mark: I prefer to have bags because if we have a bucket it will take too long to be cleaned.

Jane: How would we open it for collecting the rubbish?

Mark: We can open it from the front or from the side.

Jane: If it opens from the front it will be good for the cleaners to pick it up, if it opens from the side passengers sitting beside the bin would have to move to let other people put the rubbish in.

Mark: In both cases the bin is too close to the passengers for the cleaners to pick it up, it will be disgusting.

--- New customer requirement: the number of units should be limited

Mark: None of the three positions will be useful, as there will be too many units to be installed.

Jane: This is making me think that we should have an automatic system that packages and collects the rubbish instead of manual.

Mark: In a train a bin is used for peel, paper... not big items. We can design a small bin.

Jane: If it is small, we should place the bin under the window in a way that opens from the top.

Mark: Is there enough space?

Jane: Yes, there is, we can hide it inside the wall and the collection will be automatic.

Here we omit the rest of the dialogue, in which the system is then designed with bins under the window and bags with plastic zippers fixed on a conveyor belt that passes through the whole compartment, and shifts every time a bin is used; when a bag is full it gets replaced. 
In this dialogue, we can observe that there are a number of subtasks to be solved, such as where the bin is placed, how it should be shaped, and how rubbish should be stored and collected. All of them are interrelated, and information about one subtask informs a solution for a different subtask. Let us focus on where should we place the bin; in Figure 5 we show the arguments for the identification of different alternatives. In the initial part of the dialogue, three options are formulated, where each of them is formed after another is deemed not acceptable. The last option (along the aisle) is challenged by Jane but remains an open alternative until Mark dismisses all three with the two arguments. Mark also shares some information about the behavior of the cleaners that informs Jane's option of positioning it under the seats. A fourth position is considered, also informed by some information about the usage of bins in the train, and finally this is the one chosen.

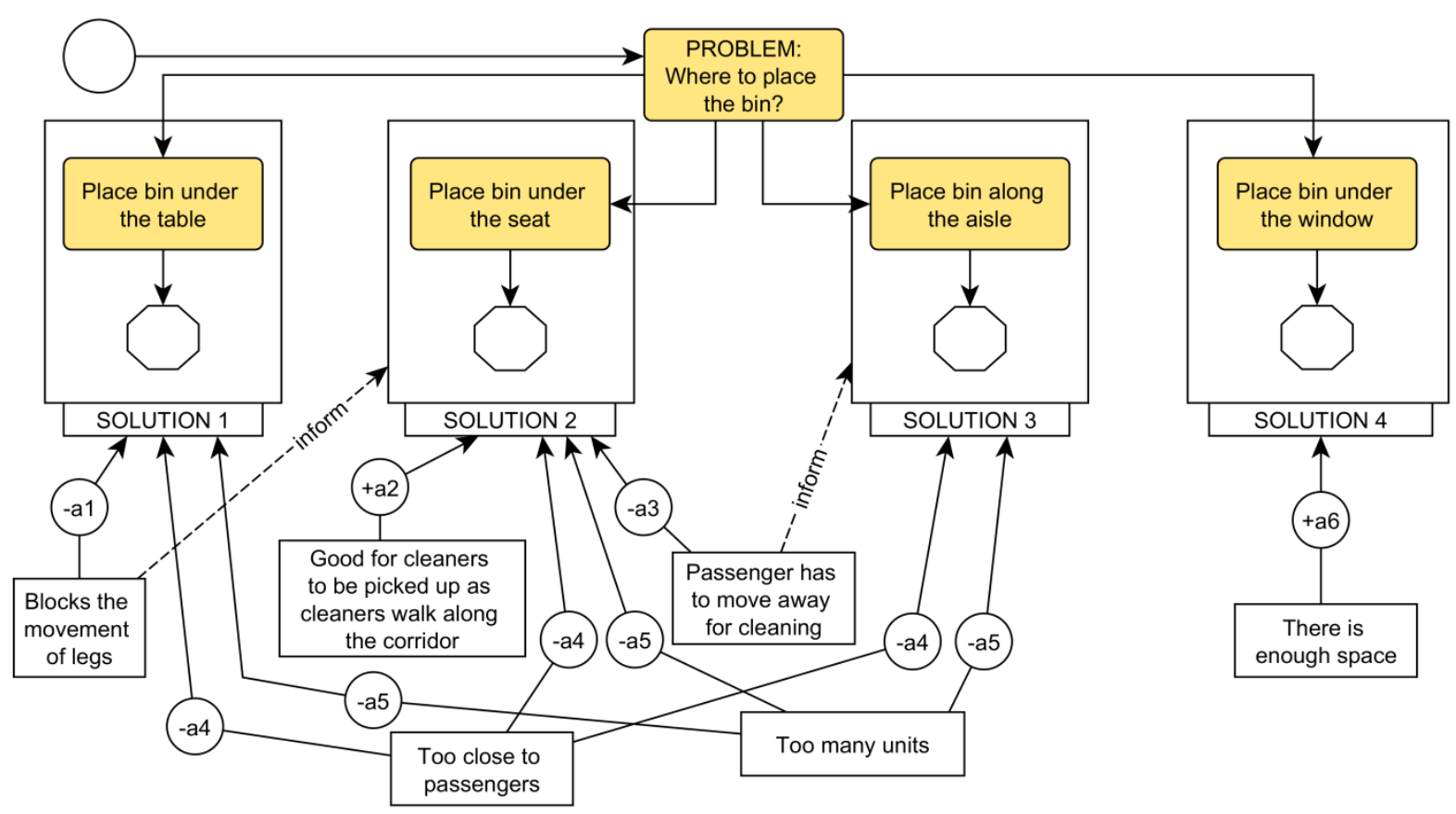

Figure 4: Revision of position of the bins

Each sub-task functions as a criterion to shape the design of the bin. However, in many design scenarios, we might encounter deliberations in which sub-issues are advanced and further discussed before reaching a conclusion for the main task. Assume for example that in order to have a bin under the window, a specific material is required that may not be available. A sub-issue is then opened to identify how to retrieve the required material in order for the solution to be feasible. Differently from the car example, however, here the new options are combinations of options, such as having a bin in four different positions each of those with or without bags, and that may open from top or the side. Similar to the car and the disaster examples, the new options are guided by the new information shared during the dialogue which may for example come from an information-seeking process (such as the type of rubbish used). Since new information may come from a change of circumstances, in this case we have an additional requirement acting as a main con argument that eliminates all the existing alternatives.

\subsection{The COSTAR Example}


The Hubble Space Telescope was launched into orbit around the earth in 1990 and remains in operation. Once it was in orbit and transmitting pictures back to NASA, the blurry images showed that a main mirror aberration made Hubble ineffective for its intended purpose of providing images of distant stars and galaxies. The project was judged to be a failure and the discussion shifted to an investigation aimed at finding out what was wrong.

The astronomers suspected something was wrong with the mirror. Next there was an investigation that concluded that the failure was a so-called "spherical aberration". The primary mirror and the secondary mirror needed to focus exactly at the same location in order to produce a clear image, and this was not happening. This aberration could not be fixed from earth. A device called COSTAR (Corrective Optics Space Telescope Axial Replacement) was built that could correct the aberration once installed in Hubble. In 1993, a team of five astronauts inserted COSTAR into the orbiting Hubble in order to correct the aberration.

Below is a step-by-step summary of the ten points in the process of deliberation that took place at NASA after Hubble had been judged as a failure. ${ }^{1}$ After each of the points (in italics) we have inserted interpretive remarks on the stages of the dialogue.

1. A NASA board of inquiry found that the cause of the problem was that a few missing chips of paint had thrown off the laser-guided measuring tool used to shape the mirror.

Now they had located the problem more precisely, the next step was to look for a solution.

2. A panel was then formed to find a solution.

Note that the people on this panel were a somewhat different group from the people who worked on the investigation finding the problem. Nevertheless from the point of view of deliberation dialogue, the structure of the dialogue holds together as a coherent unity because both groups were engaging in carrying on a continuing problem solving deliberation dialogue aimed at a common goal of getting the Hubble to work.

3. At the next point, another committee was convened, a so-called blue ribbon committee, where the members brainstormed about all kinds of possible solutions, including bringing the telescope down.

This stage was pure problem solving deliberation. Note that the agents who took part were different from the prior group, but contained some of the same members.

4. They thought up all kinds of ways to fix the telescope, but none of them seemed to be any good. They finally came to the solution that the best way to correct the problem was to put small corrective mirrors in front of the cameras and other instruments.

This became another sub-goal (putting the mirrors in front of the cameras), which however then led to no option, so they rolled back to a different solution. At this point they came to a general conclusion about the necessary means to solve the problem, but they still had not solved the problem of how to actually fix the aberration.

5. They concluded that there was no way to make the needed corrections while the Hubble remained in space. Building another telescope to replace Hubble would be too costly, and it was not feasible to try to bring Hubble back to earth and repair it.

At this point they drew the conclusion that they needed some way of repairing the mirror alignment in Hubble. But they still had the problem of how to do that.

6. Jim Crocker came up with an imaginative solution based on an argument from analogy. While he was taking a shower, he noticed that the showerhead was on a sliding rod, and that the head pops up and down as you enter or exit the shower. He drew the conclusion that if the team could pack the mirrors into a robotic arm, and put that arm into a casing, little mirrors could flip out in front of each of the other instruments to correct the misalignments. 
At this point they had a concrete proposal that showed promise of solving the prior problem of correcting the misalignment.

7. At the next step, NASA agreed to go ahead with trying to implement this solution by means of devising an instrument called COSTAR, which had arms that would flip out, the way Jim Crocker's showerhead did.

At this point they made the decision to go ahead with Crocker's proposal.

8. The next step was to build this device.

This is the engineering part of implementing the device needed to make the solution work.

9. The next step was for the space shuttle Endeavor to set off on its mission to service the Hubble in space. When the crew arrived at the Hubble, after two days, they had to do five spacewalks to do the repairs. On the third walk they needed to replace Hubble's field camera. As astronaut Story Musgrave removed the camera's cover, he had to expose and relocate the camera's delicate mirror, an operation that took two hours. The final repair for astronaut Kathryn Thornton was to pull out the old instrument and insert COSTAR with its new corrective mirrors. It took two hours to get the instrument fitted exactly into place as planned.

This step was really a sequence of actions carried out by the five members of the repair team. There is no need to go into the details here, but there had to be much problem solving. 10. When the scientists returned to earth they viewed the images they saw produced on their screens by the Hubble telescope. The images were now clear, and the small points of light representing stars in space that were not visible before now showed up very clearly until the whole screen was filled with them. The scientists cheered. Success!

This was the closing stage of the problem solving activity tracking back to the opening stage where the problem that the Hubble was not working was encountered. At this point the problem was solved. Hubble now worked the way it was intended to from the beginning.

This example illustrates what we presume to be a feature common to many examples of deliberation dialogue. If you track it back, the deliberations began with the decision of NASA to go ahead and build Hubble and launch it as a device to be used for specific scientific purposes. So they actually carried out this project but then when the results came to be tested, it was found to be a failure, which led to the NASA investigation that concluded that the failure was due to a spherical aberration. This led to further discussions about how this problem could be fixed. As always, the options were limited by cost and other factors. So then, during the course of this deliberation dialogue, a secondary dialogue was initiated that was specifically a problem solving dialogue. They realized they had a problem, and then they searched for a solution. Once they had a proposal for solving the problem, they went ahead and implemented that, and ultimately it worked. So here from the dialectical point of view we can track back from the start point of the problem solving dialogue to a prior decision making dialogue concerned with building and launching Hubble.

There was deliberation even prior to point 1, then there was the failure. This failure posed a problem for NASA and for all involved, but then there was the NASA investigation of the failure, which diagnosed the spherical aberration as the specific problem to be examined. Once the sequence got to this point, it became a problem solving dialogue addressed to solving this problem of correcting the defect (if possible). Ultimately what made it possible was the building and installation of COSTAR. Tracking the dialogue sequence from points 1 to 10 then, it was a problem solving dialogue. But along the way, decisions had to be made. For example when the proposal had been put forward, there had to be a decision whether to go along with the COSTAR project or not.

Figure 5 gives an outline of the sequence of problem solving argumentation in the COSTAR example as it goes through the characteristic three stages of an argumentative dialogue similar to the model of McBurney and Parsons (2002). We start at the top with the 
circular node that indicates the start point of the procedure. The parts of the sequence that are included in opening stage are indicated by the dashed lines at the top left. As indicated in Figure 5, the problem is identified and formulated in a general way, but also formulated more precisely as new evidence comes into the deliberations through the observation that the Hubble telescope is not working properly because of paint chips thrown off by the laserguided measuring tool used to shape the mirror.

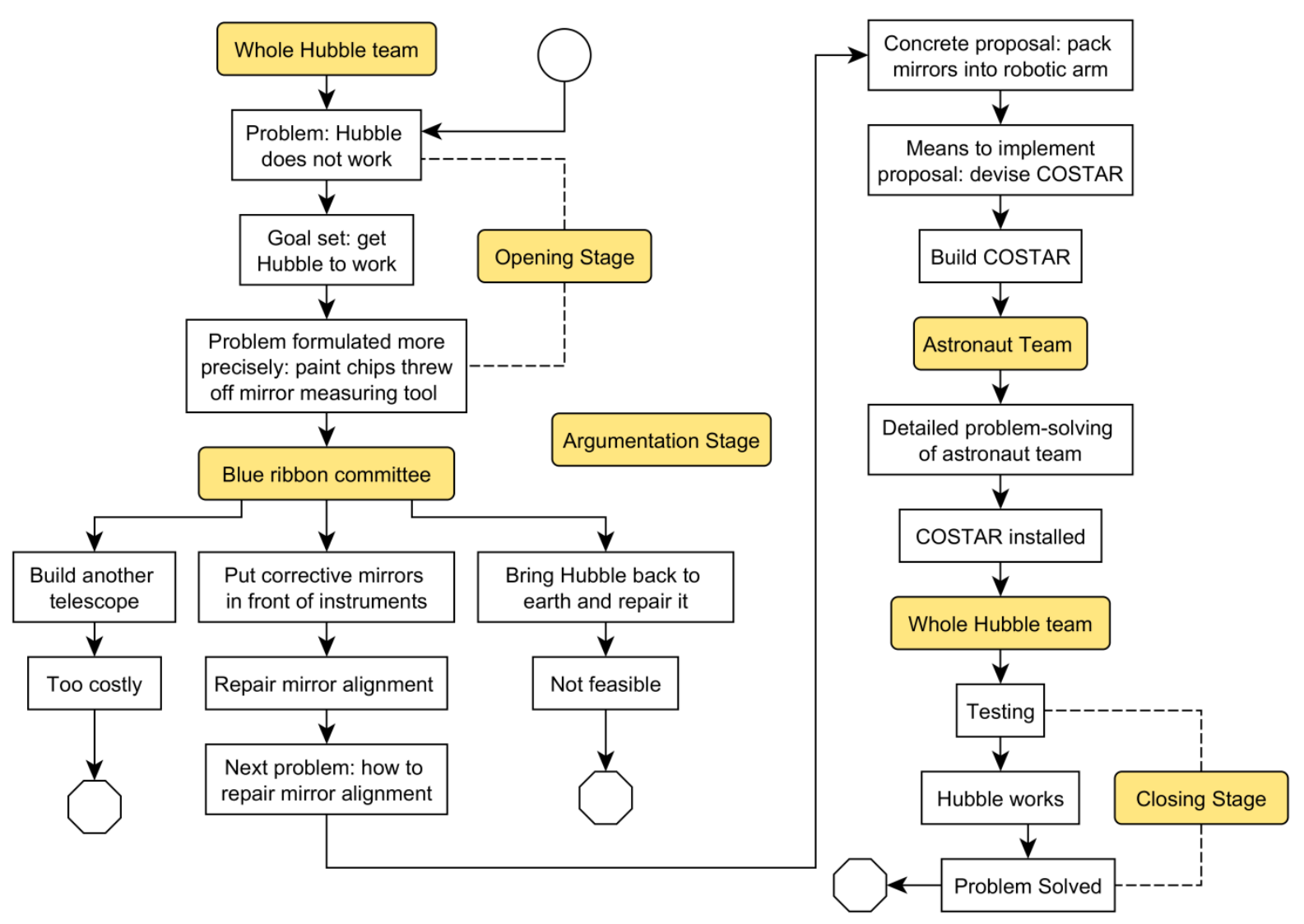

Figure 5: Problem solving argumentation in the COSTAR Example

Once the team has identified the problem, the sequence proceeds to the argumentation stage. Notice that at this stage the deliberations begin to be carried out by a different group of agents, in this instance a blue ribbon committee that is a subcommittee of the Hubble team that has been identified as having the expertise to work on a technical solution to the problem. At this stage, three proposals are considered. The first one is to build another telescope, but the argument that doing so would be too costly leads the committee to discount this approach. The second proposal, to bring Hubble back to earth and repair it, is discounted as not feasible. The third alternative, to put corrective mirrors in front of the instruments, is selected as the best proposal of the three.

From this point, we are led in the problem solving dialogue through another sequence of actions that will ultimately lead to the solution of the problem shown at the bottom right of Figure 5. The first step in the solution was to recognize that putting corrective mirrors in front of the instruments could possibly be a means of solving the problem. Next it is recognized that this would require mirror alignment, which poses a problem of how to make the mirror alignment repair. Here we have an interesting instance where the general problem of getting the Hubble to work has led through a series of other problems to the more specific problem of 
how to repair the mirror alignment. This is typical of problem solving: solving a general problem leads to a sequence in which many other problems are posed that show the way forward once they have been solved, to an ultimate course of action that will in the end provide a solution.

Notice again along the right column of Figure 5 that the participants in the dialogue shifts from the participants in the blue ribbon committee to the participants in the astronaut team which was ultimately tasked to install COSTAR. Once COSTAR had been successfully built and installed in Hubble, the Hubble team was reassembled to test the device to see whether it worked by looking at their computer screens as the device was activated. This part of the sequence is here classified as making up the closing stage, where the problem was solved.

\subsection{The education example}

An important type of dialogue closely related to deliberation is advising dialogue. Advising dialogue was involved in all of the examples of deliberation we looked at. In the printer example, Anna not only gave Brian advice on how the black line could be removed, but in the end she wound up helping him to remove it by the method she recommended. Brian found the advice he needed to get the solution to this problem by searching on the Internet. He eventually got what he took to be the best advice, after much searching, once he found the manufacturer's website (Walton, 2015, 168-171).

This example, a one-page article in The Economist (August 1, 2015, p 9) poses a problem by describing a given situation, and offers advice on how to solve the problem. The advice is based on weighing pro and con arguments. The given situation is described as follows:

"Education in most of the developing world is shocking. Half of children in South Asia and a third of those in Africa who complete four years of schooling cannot read properly. In India 60\% of 6 to 14-year-olds cannot read at the level of a child who has finished two years of schooling."

The article discusses two potential solutions to the problem: government schools and private education. The article then makes a number of factual statements, backed up by statistical claims, leading to the conclusion that government education has failed:

"In a survey of rural Indian schools, a quarter of teachers were absent. In Africa the World Bank found teacher-absenteeism rates of 15-25\%. Pakistan recently discovered that it had over 8,000 nonexistent state schools, $17 \%$ of the total. Sierra Leone spotted 6,000 'ghost teachers', nearly a fifth the number on the state payroll."

The article continues to state that part of the problem is the powerful teachers' unions who see the jobs as sinecures, and the state education budget as a revenue stream. The article states that for these reasons, and with the growth of the economy and jobs requiring education, there has been a boom in private schools. Since the unions are fearsome enemies, the governments leave them to run the schools.

This case is not an instance of deliberation dialogue, because The Economist, or at least the author of the article who wrote it for The Economist is not in a position to make the decision for private or public education, one way or the other. This is in contrast to the car buying example, where the participants in the dialogue are in the position of actually making 
the decision. It is an instance of advising dialogue, where one party offers advice to the other about a problem faced by the second party (Grasso, 2015). The advising party enters into the deliberation, examines the pros and cons on both sides, and presents a solution that can be used by the other parties making the decision. This form of article is common in The Economist, in cases where the problem is to a significant extent one of economics. The Economist typically collects the relevant facts as statistical findings and financial factors that bear on the problem and uses this data to argue for a recommended course of action that would solve the problem.

The article presents three arguments to support the furthering of private education as a solution to the problem, as represented in Figure 6a.

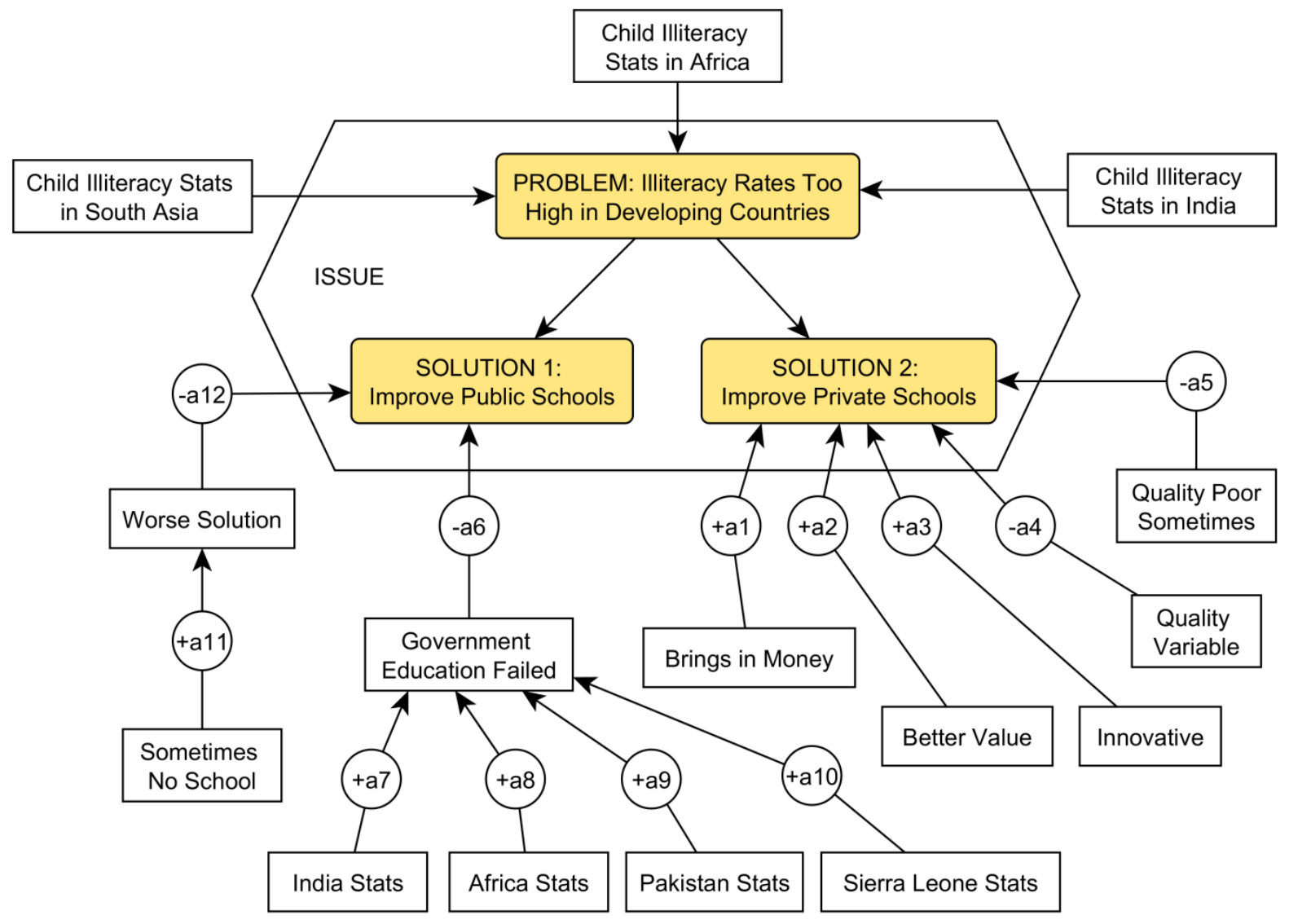

Figure 6a: Argument Map of the Education Example

First, it brings in money, not only from parents but also from investors. Second, private schools are often better value for money than state schools. Third, private schools are innovative, because they use computer technology to provide teaching materials linked to a central system that can monitor work. These arguments are shown in Figure 6a as arguments $a 1, a 2$ and $a 3$. Pro arguments are shown in round nodes containing a plus sign. Con arguments are shown in round nodes containing a minus sign.

The article does not just consider arguments for the private school solution to the problem. It also cites some arguments of critics of the private school solution. Critics argue that the quality of private teaching is variable, and sometimes poor. As a counter to this argument the article states that private school is better as an alternative than public school, which is a worse solution because it sometimes means no school at all. In conclusion, the 
article offers the advice that government should therefore be asking how to boost private education, instead of discouraging it. The action proposed is that governments should either subsidize private schools, or at least stop trying to block their attempts to move forward.

Although this example, on the surface, has the form of a monologue, it can be modeled from a normative argumentation point of view as having a dialectical (dialogue) structure, which presents all characteristics that we can identify in a typical deliberation dialogue with an open knowledge-base as discussed for example in Walton, Toniolo and Norman (2016). We will further discuss this view in Section 4. The argumentation in the text is a highly typical use of practical reasoning to offer arguments that conclude with advice in deliberations on how to solve a problem. First, the problem is described by setting out factual and statistical assertions that described an existing situation. Second, alternative solutions to the problem are discussed and arguments are brought forward as arguments pro and con each proposed solution. Solutions can be posed following the scheme for practical reasoning, highlighting reasons including values for supporting or attacking a particular solution.

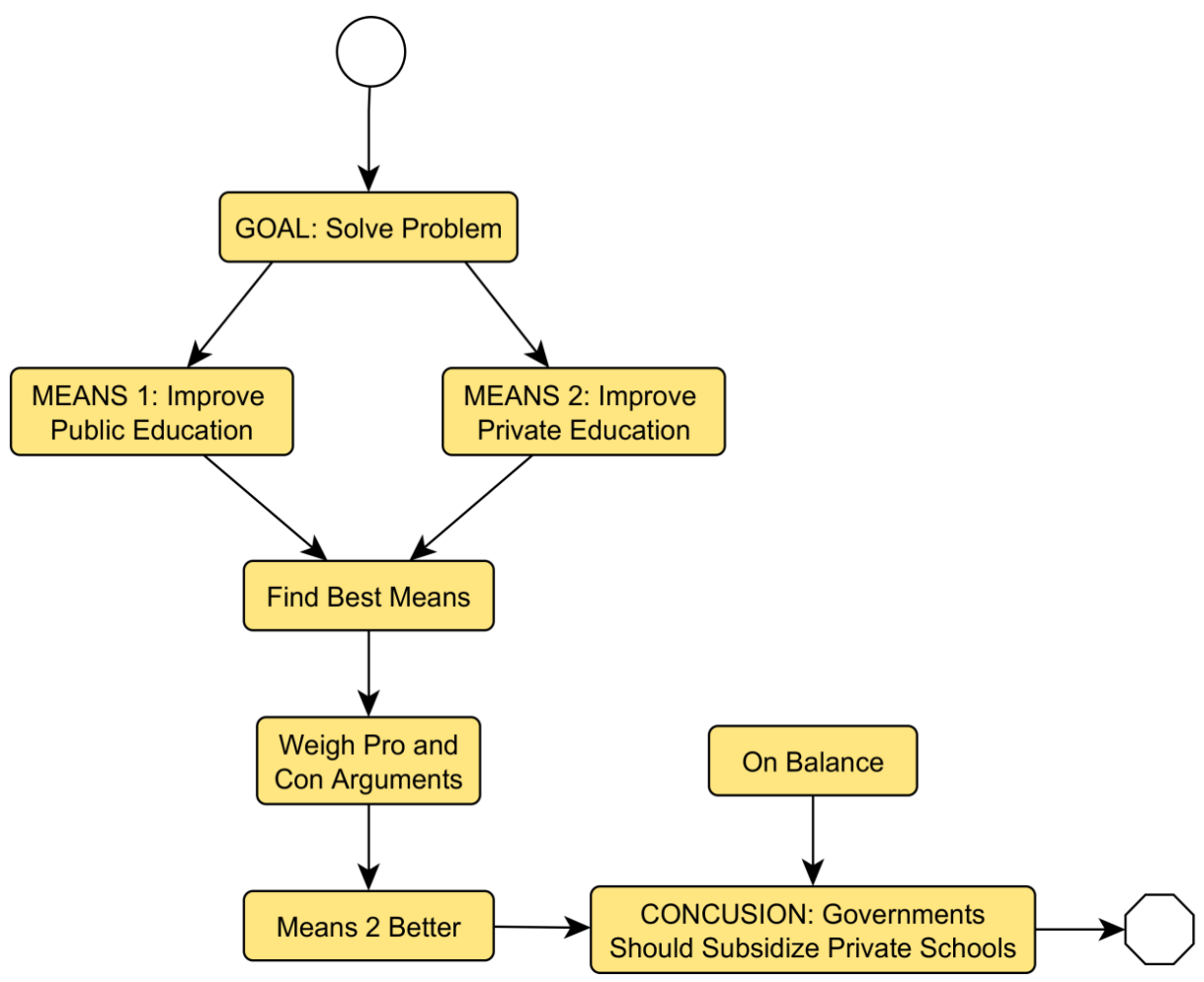

Figure 6b: Practical Reasoning in the Education Example

On balance, one proposed solution comes out to be the best one, and on this basis the sequence of argumentation going through the twelve arguments shown in Figure 6a results in the conclusion that this solution should be taken up as the recommended course of action. Figure $6 \mathrm{~b}$ shows how the procedure works in this example. The way the deliberation process is set out is shown as a sequence of practical reasoning. The goal is to solve the problem, and various means of moving forward towards solving the problem are discussed. In this instance, two means of solving the problem are discussed, improving public education or improving private education. The discussion moves forward by bringing forward arguments about which is the better of the alternative means. The next part of the procedure is a typical argumentation interval in which pro and con arguments are put forward supporting or attacking each means that is postulated. 
The circumstances pose a problem that needs to be solved. The means are to improve teaching. But the problem is how to do that. Two alternative courses of action are considered, improving public the (state) school system or improving the private schools. Arguments pro and con both options are considered. Through a sequence of argumentation of the kind shown in Figure 6a, a finding is arrived at that the one course of action is better than the other. This conclusion is put forward as the solution to the problem. The Economist article has volunteered to take part in the deliberation by offering advice on what is the best course of action based on the factual evidence presented.

\subsection{Observations}

In Section 3, we have presented a number of case studies, through which we have shown that there exists a type of deliberation called problem solving deliberation in which there is an initial problem to be solved. The initial issue states a number of options, a number of alternatives that agents are expected to deliberate upon, but this issue may be revised during deliberation according to new information or changes of circumstances. The revised alternatives are identified, guided by information pro or con existing alternatives, and they are considered relevant as long as they provide a general or partial solution to the initial problem. The issue itself may also be dropped if the circumstances of the problem are no longer verified. Alternatively, the dialogue may lead to identify sub-issues that if solved may be able to provide a viable alternative for the original issue.

The examples analyzed above using argumentation methods suggest that prior to the advent of argumentation theory, deliberation used to be modelled by calculations that tended to ignore or underemphasize the pro and con arguments that led to the ultimate decision at the closing stage of the procedure. In economics and the other social sciences decision making deliberation was typified by a cost-benefit decision procedure that emphasizes the costs and benefits of the outcome, rather than the argumentation prior to this closing stage. In moral philosophy, value-based deliberation was often studied by considering so-called trolley problems, representing a choice to be made between two or more fixed options and abstract examples of ethical decision making without considering larger and more realistic instances of every day deliberation where the issues can be more complex as discussions between the participating agents become relevant where the parties in the group of agents interact argumentatively and bring in new evidence as the case evolves. We contend that these examples suggest that argumentation theory needs to distinguish between different kinds of deliberations, and to try to see how they relate to each other.

Our hope is that this can give rise to further discussions on whether we can have different models for different kinds of deliberation, to get a better understanding of how the models and the examples can lead to a classification system that (1) defines the different kinds of deliberation dialogue and their subtypes, and (2) thereby gives us a better empirically based theory of that enables us to grasp how argumentation relates to decision making and problem solving (existing widely accepted technologies).

In particular, the examples presented show that the more flexible dynamic model of deliberation introduced in Section 2 includes at least two types, a "decision making" deliberation, where the focus is on making a decision on what to do, and a "problem solving" deliberation focussed on solving a problem, both forms of dynamic deliberation where the issue is revised during the dialogue. We now examine in more detail the difference between these two new types of dialogue that, we believe, have received less attention in the philosophy and artificial intelligence literature.

\section{Problem solving and decision making}


The examples of deliberation dialogue studied in this paper fall into two categories. Some of them are clearly examples of problem solving, such as the printer example, while others are clearly examples of decision making, such as the car buying example. The education example certainly involves deliberation, and many readers might be inclined to classify it as an instance of deliberation, because it is about what to do in a specific situation. However, we saw that it does not quite fit the deliberation model. It is best classified as an instance of advising dialogue. The bins example involves solving a number of closely related design problems, and can be classified as a problem solving type of dialogue, even though it requires the team to make a number of decisions. The COSTAR example is clearly an instance of problem solving, but as we saw, it also involved decision making at several points.

In order to better characterize problem solving and decision making deliberation, and understand their differences, we analyze established theories of agent problem solving and decision making in Artificial Intelligence. Decision theory is an interdisciplinary structure shared by many fields including economics statistics, psychology, political science, computer science and philosophy (Steele and Stefánsson, 2015). Broadly speaking it aims to provide a systematic method for assisting an agent, or group of agents, to make choices about what to do in a situation where the agent needs to, or wants to arrive at a reasonable decision, according to some standard of rationality. Decision theory (Anand, 1993) is based on the idea of expected value, which dictates that the rational procedure for making a decision is to identify all the possible alternatives, called the outcomes, and assign a numerical value, (positive or negative) to each alternative. The expected utility is calculated by multiplying the probability of the outcome and the value of the alternative (such as an amount of money). The outcome to be chosen should be the one that represents the highest expected utility, based on this calculation.

Multi-criteria decision making (MCDM) is a branch of operations research that evaluates multiple conflicting criteria in decision making, both in daily life and in settings such as business, government and medicine (Munier, Hontoria and Jiménez-Sáez, 2019). Typical criteria used, for example in analyzing a decision on what car to buy, are safety, cost and style.

A recent development in AI has been the advent of several formal argumentation systems that combine argumentation theory with MCDM (Ouerdane et al., 2011; van der Weide, 2011). Here we cite one example of such a system that illustrates how such a combination is possible. In version 4 of the Carneades Argumentation System, each of the premises of an argument are taken to represent the value of some property of each of the choices being made by using an MCDM. Weighing functions are used to assign numerical values to hard and soft constraints that figure in a given case of issue-based decision making argumentation. In the car buying example, configured in the Carneades style, as illustrated by Figures $1 \mathrm{a}$ and $1 \mathrm{~b}$, there can be hard and soft constraints. For example, when trying to decide which car to buy, a hard constraint would be the need to satisfy safety regulations (specified by law). Soft constraints are used to compute the weighted sum of properties representing the values of the decision-maker that do not denote hard constraints. A soft constraint is represented by an integer denoting the relative weight of a property by assigning it a numerical value in the range between 0 and 1 (Gordon, 2018, 766). For example, numbers representing low, medium or high values could be assigned to safety, sportiness and costliness. Once these values are assigned, argumentation schemes indicating the strength or weakness of an argument, along with consideration of which of its premises are accepted or not, are used to show which of the options should be weighted more or less highly than the others by the system. In Carneades it is the user who inputs these values and the system can 
then use argumentation to comparatively evaluate how each of the options at issue are supported by the values and the relevant factual evidence.

Let us look at a very simple example that can be used for illustration such as the one shown in Figures 1a and 1b. Note that the two premises at the bottom right of Fig. 2 state that $\mathrm{CR}$ is an expert source, and that CR says that Camry scored high in crash tests. These statements fit as premises in the simplest (heuristic) version of the scheme for argument from expert opinion. But then let us also take a look at the pro argument a6 shown at the top right. Its two premises, 'Volvo ad is a reliable source', and 'Volvo ad says Volvo is safe', go together in a linked argument fitting the scheme for argument from witness testimony to support the conclusion that Volvo is safe. The general idea is that the values can be weighed alongside the usual way that argumentation is evaluated in the Carneades Argumentation System to calculate how strongly each option in the issue as stated is supported (or not) by the factual evidence and the values of the decision-maker.

Poole and Mackworth $(2010,372)$ comment on building an automated system that gives advice to someone, stating that the builder of the system should take into account what the values of the user are. The argumentation-based decision making deliberation approach accounts for this aspect by using argumentation schemes for argument from positive consequences and argument from negative consequences, where what is positive or negative is determined by another scheme called argument from values. Decision theory works in a different way by showing that a rational agent's value for an outcome is measured by a real number representing the agent's preferences and a probability value representing the likelihood of the outcome. There is however a similarity in the process and outcomes of the two approaches, which involves a choice among a number of actions and some criteria that lead to this choice. We argue that our examples suggest the hypothesis that in addition to decision making deliberation, the type of deliberation that has received more attention in the literature, there is a second subtype: problem solving deliberation.

Klein and Iandoli $(2008,1)$, for example, define 'collaborative deliberation' as an activity where "groups of people (1) identify possible solutions for a problem, (2) evaluate these alternatives, and (3) select the solution(s) that best meet their needs". It is clear from the wording of this definition, including the words 'problem' and 'solution', that they see deliberation as being a species of (or at least as involving) problem solving. Deliberation in their sense is a type of cooperative problem solving argumentation by multiple agents who typically engage in large-scale interactions using argumentation in the format of a dialogue structure. Klein and Iandoli $(2008,1)$ also note that while computational tools have been successful in enabling mass knowledge sharing and communication of ideas, such communication has become notorious for having massive numbers of low-quality posts, especially on controversial issues. Indeed as Klein $(2017,1)$ notes, deliberation processes which have changed little over the ages typically work by having powerful players craft policies behind the scenes and then try to get wider support for their preferred options by looking for arguments that will be persuasive to the crowd.

The terms decision making and problem solving are already widely used in many disciplines, in ways that indicate they are generally considered to be similar to applications of our deliberation theory.

Some general remarks provide an entry point into the discussion. In engineering, a starting point for problem solving is the failure of the process or product requiring a correction to prevent future failures. In psychology, problem solving is treated as a mental process, whereas in computer science, it is treated as a computerized procedure for searching to find a means to solve a problem. Goal-directed practical reasoning is clearly central to the computer science meaning. But both fields have the idea that there is a procedure of problem 
finding prior to the procedure of solving the problem. In general it is distinctive of problem solving that there is a goal to be reached, but not of decision making.

Bransford and Stein (1993) have set out what they called the problem solving cycle involving several distinct stages: recognize the problem, define the problem, try to find a strategy to fix it, figure out what can be done with the resources at hand, monitor one's progress, and evaluate the proposed solution that has been arrived at. This appears to be different from decision making. Among the techniques recognized as strategies for problem solving are the following: using a solution based on a prior solution to an analogous problem, brainstorming by discussing proposed solutions, moving forward towards a solution through a sequence of actions where means are connected to goals, and testing proposed solutions to try to determine which is the best one.

Russell and Norvig $(1995,53)$ characterize a problem for a problem solving agent as a goal and a set of means for achieving the goal. Planning can be seen as a sequence of steps to solve the problem. This is an interesting way to proceed from an argumentation viewpoint, because it essentially defines what a problem is in terms of the argumentation scheme for practical reasoning. On their account, intelligent agents of this kind are capable of goal formulation and problem formulation and the problem is that an agent has to find out which actions in a sequence of actions will get to a goal state that it has formulated. In well-defined problems (Russell and Norvig, 1995, 60), there is an initial state that the agent knows itself to be in, a set of possible actions available to the agent, and a path, defined as a sequence of actions leading to the goal state. The state space is formed by all reachable states from the initial state defined by all possible actions. In addition, there is a global test that the agent can apply to any single state description, essentially a proposition, to determine if it is a goal state. Another component of problem solving is the cost of a specific path, represented as the sum of costs of individual actions along the path. Problem solving is carried out by searching through alternative paths in the state space. On the description of problem solving set out by Russell and Norvig $(1995,55)$ a problem is solved when the problem solving rational agent decides what to do by finding sequences of action that lead to desirable states. Similarly, Poole and Mackworth $(2010,71)$ define problem solving as a search procedure that an intelligent agent uses to achieve its goals by finding a path that is essentially a sequence of actions that will achieve its goal before it can take the appropriate actions to achieve that goal. An alternative approach is that of Hierarchical Task Networks (HTN) (Erol et al., 1995). Interesting in this approach is the representation of an objective as a set of subtasks. The primitive actions are placed at a lower level of the hierarchy, while complex actions composed of primitive actions are placed in higher levels. The solution of the problem is formed by refining downwards from the goal until a sequence of primitive actions is found.

Decision making is a term widely used in different disciplines, such as economics, computer science and psychology (Steele and Stefánsson, 2015). The bounded rationality model is regarded as especially important. This is the principle for closing off the decision making procedure when it reaches a reasonably acceptable or good enough solution, rather than continuing the search for a maximizing solution (a search which itself has costs).

Related to this, the widely established decision theory, or the theory of choice, previously introduced, is said to have two branches. Normative decision theory gives advice on how an agent should arrive at the best decision based on the agent's preferences and values.

Descriptive decision theory studies how agents actually make decisions, even if they arrive at a decision not supported by rational calculations.

In a chapter (Chapter 9) on planning under uncertainty Poole and Mackworth (2010) give an account of decision making meant to be applied in artificial intelligence that might help clarify how they take it to be different from problem solving. They write (Poole and Mackworth, 2010, 373) that what a rational agent decides to do should depend on its 
preferences. In the argumentation model of deliberation dialogue, the agents engaging in deliberation frequently have to set their own preferences aside in order to arrive at a decision with other agents in the decision making group that have different interests and different personal preferences. They write (Poole and Mackworth, 2010, 372) that when an agent must reason under uncertainty the designer of the agent must specify trade-offs between different outcomes to take into account whether these outcomes are good or bad, or even disastrous.

One may argue that decision and problem solving theories represent forms of reasoning but do not present the typical dialectical (dialogue) structure, particularly if only a single agent is involved, as in the case of the printer or the education examples. What we mean by the term 'dialectical' in this paper, as applied to deliberation, problem solving and decision making, has much in common with an earlier theory. Churchman (1971), Mason (1969) and Mitroff and Kilmann (1978) advanced a theory of dialectical problem solving and applied it to situations in which a manager in a business environment constantly faces problems where there is a need for the best available evidence and limited time for taking action in order for an organization to survive. Their method works by setting up two antithetical views that are in direct conflict with each other on how to form policies and solve problems. This dialectical framework is seen as a dialogue exchange between, in the simplest case, two parties in which the one party challenges views taken by the other party as basic and reasonable assumptions. The purpose is to assist the problem solver to operate in a turbulent environment where conditions are quickly changing in order to convert a problematic situation into an opportunity (Mitroff, Emshoff and Kilmann, 1979, 583-584). This dialectical theory of problem solving was meant to be applied in complex situations in business involving the formation of different groups among stakeholders who engage in dialectical debate to find group policies.

This earlier theory of dialectical problem solving works by formulating different views on a particular strategic policy question so that the decision-maker can compare and contrast the pro and con arguments on both sides in order to see how they interact and thereby get a deeper view of aspects of the problem and proposals for its solution that might otherwise not be considered at all. This earlier view of dialectical problem solving was not based on argumentation theory, but arose out of a practical need to assist operations research on strategic problem solving in business and other practical undertakings. It has much in common however with the recent argumentation-based theories of deliberation. We have shown in this paper how this dialectical approach to problem solving, decision making and deliberation can even apply to the case of a single agent (e.g., in the printer example) who is confronted with different potential solutions to a practical problem.

\subsection{Deliberation in problem solving and in decision making}

By reviewing existing theories of decision making and problem solving, we are now confronted with two general questions, how our formal theory of deliberation fits within these approaches, and how to build criteria for distinguishing between decision making and problem solving as species of deliberation dialogue.

We are interested in representing decision making in complex, uncertain situations where agents may have different interests, goals, and different perhaps incomplete views of the world. Agents may act on behalf of users, or interact with users in the process. In our examples, decisions on what to do or how to act are underpinned by argumentation, where the evaluation of pros/cons is fundamental for a solution to be adopted. This feature permits argumentation-based systems to represent reasoning in a way that is close to human 
understanding (Mercier and Sperber, 2011) and is especially important when developing systems where humans and autonomous agents collaborate in deciding what to do.

From an argumentation point of view, one important difference between decision making and problem solving as types of deliberative dialogues is the opening stage. With decision making, it is clear that the opening stage is where an agent or group of agents confront a choice on what to do, or how best to move ahead, in a given set of circumstances where there are several alternatives, at least two, and the agents have to choose the one or the other. In some cases one of the alternatives is simply doing nothing. In contrast, in the problem solving type of dialogue, at the opening stage the agents have to define the problem, or in other words to determine what the problem is. This seems easy in some cases, but is less obvious in others. In the printer example, the problem is simple. Brian's printer is not working, and that is a problem, because Brian needs it. In other examples, we have drawn a distinction between a general problem and a particular problem. Poole and Mackworth (2010, 72) draw a distinction between general problems and specific problems when they write that in problem solving, humans most often do not tend to solve general problems, and they concentrate on specific instances which they may know more about. They suggest that for this reason the computational problem solving agent should exploit knowledge gained from solving problems in specific cases to guide them to a solution to a general problem.

In the COSTAR example, it certainly was the general problem that the Hubble was not working properly that launched the problem solving dialogue. But further investigation, undertaken to determine more specifically what the specific problem was, led to the cause of the failure, the spherical aberration in the operation of the mirror.

In deliberation dialogue, at the opening stage an agent or group of agents confront a choice on what to do in a given set of circumstances where there are various alternatives. Decision making, if you view it in an argumentation perspective as a species of deliberation dialogue, is the representation of the set of alternatives in such a manner that the preferences of the agents can be brought to bear on deciding which is the best choice based on the knowledge that the agents have about the likely consequences of each alternative, the communal goals of the dialogue, and the individual preferences of each of the agents.

Suppose as a thought experiment that the example of Brian and his printer in Section 3.2 is modified so that Brian did not ask for any help or get any help from the Internet or his wife. He solved the problem by thinking his way through it to a solution all by himself. Can this kind of solitary problem solving still be called deliberation? And can it be called problem solving in the sense the expression is used in this paper? Is it an instance of deliberation dialogue?

The first thing is that the revised example does not really seem to be a dialogue at all. So the classical description of problem solving given in the Artificial Intelligence text books already model this procedure of problem solving as a form of reasoning with a graph structure. It is problem solving, but does not require a dialogue structure with speech acts being put forward by two parties arguing the pros and cons at each step. So it is not really deliberation in our sense of the term which requires modelling deliberation as a dialogue between agents who are putting forward speech acts in order to communicate with each other to try to solve a problem or deliberate on what to do that way. But we could still call this solitary problem solving, if Bob is mumbling to himself, conducting an internal dialogue by looking at the arguments on both sides as he goes from one step to the next in his problem solving activity.

Taking this approach, problem solving is also a type of deliberation dialogue, because it also at the opening stage must posit a choice, a set of alternatives, on what to do in a given set of circumstances. But it is a special type of deliberation dialogue because most importantly, it requires an identification of a problem to be solved in these circumstances. 
Whether something is a problem depends on what the goals of the agent are. Problem solving is all about practical reasoning, and typically the problem is that goal of the agent, or some goal of a group of agents, cannot be carried out because of the circumstances. Very often, the problem is that something does not work the way it is supposed to, given the need or some sort of quality agents have. A solution to the problem is some sequence of actions that the agents can carry out that will make the thing work. The dialogue is closed either when a solution is found, or when it is found that it is not possible to get a solution with the available resources, depending on combined factors of time and cost. Or there may be other reasons for failure. For example, it might be found that it is not possible to get a solution without defeating some other goal that the agent has, which perhaps even has priority over the other goal. It might also be found that there are additional subgoals that have to be resolved first for a general solution to be feasible.

Put in this abstract way, the distinction between problem solving and decision making is amenable to clarification by fitting the two subtypes of dialogue under the general category of deliberation dialogue. It is an attractive feature of our theory of deliberation as a dialogue structure that it includes the two types of deliberation, problem solving and decision making. Some examples clearly fall into the one category and some into the other, even though in some cases it would appear to be quite possible for the two subtypes of dialogue to be mixed in together. A case in point would be a typical example where a group of agents is trying to engage in decision making, and seems to be moving towards a decision for one particular option, but then encounters a problem with implementing this option, given the circumstances and the limits of what is currently possible. In cases of this sort, the problem solving dialogue would be embedded in the ongoing decision making dialogue as part of it. This was illustrated especially well by the COSTAR example. This is another feature which is useful to support our theory about how problem solving and decision making are related.

The same can be said for classical decision theory. Argumentation applies in multiagent cases where it is helpful to bring in pro and con arguments that act as reasons for or against a proposal or a course of action being considered. Once again, in the revised printer example, we can have a solitary agent making a choice between two actions or between several alternative courses of action without taking into account the pro and con arguments used to support or attack the acceptability of one choice or another in a set of alternatives. The costs and benefits of each alternative can be calculated using classical decision making models, and this does not have to be called deliberation in the sense we use the term, referring to the communicative exchange of speech acts in a dialogue structure with goals, agents and protocols. However, it could be useful in some cases, we are suggesting, to take into account the pro and con arguments relevant to the alternatives being considered, in addition to such factors as costs and benefits that have been calculated. In this way, the argumentation model with its formal deliberation structure could provide a resource used to extend the procedure to examples of decision making in which two or more agents are trying to better (or more deeply) find out what to do by exchanging arguments.

Having explored in more detail the differences between problem solving and decision making deliberation, we now focus our attention to how philosophical studies of deliberation have influenced the development and use of computational deliberation. We consider existing computational models of deliberation dialogue and discuss what general characteristics are still required in light of the analysis of the above examples.

\section{Computational models of deliberation}


In this paper, we argue that although deliberation has often been modelled as a decision on whether to do something or not, there are some other forms of deliberation that must be considered. In Artificial Intelligence, this is especially important as it influences the way deliberation dialogue models are developed and it changes the way a system may be designed. Computational deliberation models of dialogue have been influenced by the seminal work of McBurney et al. (2007) with respect to the dialogue protocol and by the necessary work of Atkinson and Bench-Capon (Atkinson, 2005; Atkinson and Bench-Capon, 2007) with respect to the underpinning computational representation of the practical reasoning scheme (as presented in Section 2). The latter work is specifically focussed on modelling the available arguments on what to do so that a choice can be made according to the value ordering of the participants or an audience. Nevertheless, we are interested in the different types of examples used in this work and how they are related to our theory of deliberation. Atkinson (2005) uses an example of a deliberation on whether UK should go to war with Iraq. This is the kind of deliberation that maintains status unchanged (should we or should we not go to war). The model of practical reasoning underpinning this work has then been used as model for collaborative dialogue on what to do (Black and Atkinson, 2009) with the aim to decide on a medical treatment for a patient where a number of options are presented and discussed, and later to underpin a model of deliberation (Black and Atkinson, 2011). There is a distinction between the former and the latter two examples that corresponds to a distinction between the first two types of deliberation we introduced in this paper: the first represents a dilemma-like deliberation, where there is a choice between two opposed options, and the decision making type where different options are evaluated according to their feasibility, convenience etc. Interestingly, we observe that this is similar to the influence that computational models of persuasion dialogue have had in deliberative dialogue. The studies in persuasion models, such as the one presented by Prakken (2005), have been used to propose dialogue protocols that could fit deliberation types, for example by Kok et al. (2011). Initially, the type of examples used were yes-no questions in the form of a dilemma, such as "Should we invade Iraq? Should we go for pizza?". However, later examples have moved to a decision making type of question, such as "Where should we go for dinner? What can be done to stop the Iraqi's war?" This shift in type of example, suggests a shift from dilemmalike deliberation to decision making deliberation. The examples where many actions or options are evaluated according to the agent's view of the problem or of the world, and their pros and cons have characteristics of a decision making deliberation where the options available for discussion to the agents are identified at the opening stage. While iterations through reformulation of the alternatives and deliberation is possible in some computational frameworks such as that of Atkinson and Bench-Capon (2007), we believe that this is a key difference between static and dynamic decision making deliberation. This feature should be underpinned by additional dialogue protocol elements to facilitate the identification and integration of reformulation of alternatives and revision of the issues. Of particular interest in this work is how the information acquired and shared during dialogue influences and directs the search for new alternatives available for discussion which raises the question of when and how to revise the issue.

The second distinction that we draw in this paper is that between problem solving deliberation and decision making deliberation, which is also noticeable in existing deliberation systems. In the models of Black and Atkinson (2011) and Kok et al. (2011), the aim is to decide about an action, and an agent's goal is achieved by one single transition enabled by the action; i.e. that from the initial state of the world leads to a state where such goal is satisfied. However, agents more often need to perform a sequence of actions to achieve a goal. Medellin-Gasque et al. (2011), building upon the practical reasoning scheme proposed by Atkinson and Bench-Capon (2007), have explored ways to consider planning as 
a sequence of actions to achieve a goal. Similarly, Toniolo et al. (2012) have presented a deliberation model that focusses on constructing plans between agents that have interdependent goals. These two approaches aim to employ deliberation for problem solving, presenting a full dialogue protocol. There are however other examples where argumentationbased practical reasoning is also employed for problem solving but are out of the scope of this work, as deliberation dialogue is not considered. All these examples show that there has been a shift from dilemma-like deliberation, to decision making, to problem solving deliberation. This shift, however, did not correspond to a shift in the type of deliberation protocols used. The main shift noticed in the literature was that from persuasion protocols being adapted to include moves related to actions or options.

There is a question raised here as to whether existing models, which very well represented the dilemma type of deliberation, are flexible enough to model the latter type of problems when we consider other alternatives that may come to play during the dialogue. We have already shown that the practical reasoning argumentation scheme that underpins the dialogue proposed by Black and Atkinson (2009) for example is adequate to pose a problem in the problem solving deliberation. The examples that we presented in this paper, however, suggest that there is a need for a more general account of different types of deliberation dialogue. Better understanding of characteristics such as issue revision, action sequences, sub-goals and new information is particularly important for computational models of deliberation that are typically derived from the dilemma-like deliberation and then directly applied to the decision making and problem solving types of deliberation. In particular, we suggest that the dynamic approach to deliberation is characterised by the revision of the issue made necessary by the arising of new circumstances. Since our examples show that this revision is a key difference, we now present the characteristics of such revision and how may we proceed to formulate this in a framework for deliberation.

\subsection{When does the revision occur?}

As we have seen in our examples, the introduction of new information during the dialogue often causes a revision of the issue. This is because that new information highlights alternatives that were not possible before, either by falsifying previous information or highlighting new circumstances previously unknown to the participants. New information may come from different sources. In the printer example, the observation of consequences of execution of actions may lead to the need for new alternatives. In the gelato example, new information is due to sensing the environment and hence changing the circumstances. In the disaster example, new circumstances are discovered through new information shared during dialogue. Similarly, in the car example, new options are identified through values shared during dialogue followed by an information-seeking process. Representing different methods to acquire new information may require different models in artificial deliberation for example through inquiry dialogue (e.g. Black and Hunter, 2009) or sensing (Tang et al, 2009). However, the introduction of new information results in a fundamental difference between dynamic versus static deliberation.

In previous work (Walton, Toniolo and Norman, 2016), we discussed the possibility of introducing a new speech act, disclose(Arg), to allow for agents to volunteer new information into the dialogue. The MHP model allows for a 'revise' phase in which agents may propose changes to the issue as well as to existing facts. This is only permitted through a propose speech act with no changes in the commitment store. However, a change of circumstances often requires new information to be asserted, rather than proposed. Our previous extended model (Walton, Toniolo and Norman, 2016) aimed at permitting agents to assert information about new circumstances during the dialogue. We showed for example that 
the new speech act permits agents to more effectively discuss and resolve conflicts between interdependent plans in scenarios similar to those of the disaster response. While this is a change at the protocol level of dialogue, it must be underpinned by a method to revise the set of alternatives in relation to new information.

There is also the problem of how to revise the knowledge-base but in this paper we focus on the effects on the dialogue. In some existing argumentation systems for multiagent dialogue, all the alternative feasible actions are constructed at the beginning of the dialogue or in an appropriate reformulation of the problem, and then filtered out through critiques during dialogue. When participants have different expertise, such as in the model of Black and Atkinson (2009), alternatives may not be known by all the agents at the outset but each agent will know a subset of all possible actions. However, in problem solving, when a sequence of actions constitutes a solution, agents may be aware of some solutions, but it might not be feasible to construct all solutions possible in advance, hence agents should be able to construct new alternatives guided by the new information shared and collected during the dialogue. The new information may result in changes in the set of options available for agents to discuss and relationships among arguments. In parallel to the revision of the set of alternatives, in problem solving deliberation new information exchanged by the participants in the dialogue might highlight the need for revising the issue to focus on sub-issues. A primary objective may have many sub-goals, which in turn may be achieved by many alternative actions. A dialogue about a primary issue may reveal that there are sub-issues to be resolved. However, in problem solving relevant sub-issues are not explicitly stated at the opening phase but only considered if there are conflicts with the solution proposed. Consider for example the case in which there are two solutions to a problem formed by different sequences of actions. Assume that in one of the options, all actions but one are revealed to be agreeable by all participants. In the disaster example, participants would potentially agree to stop the water supply, but one agent is then required to find an alternative location for the field hospital. The dialogue may then turn into finding alternatives for the subtask which is only revealed because part of a solution currently under discussion leads to conflicts in the achievement of the primary goal. There may be other sub-issues with respect to the other solution that are not revealed in the dialogue unless the first solution does not lead to agreement.

An additional open question is: when is the right time to consider a new option and revise the issue? In the disaster response example, we assumed that the new alternative is formed when there is no agreement on the option under discussion. We may be instead in a position of having no further choices as in the case of the bin example. Both of those are extreme cases in which an alternative is needed. This is also the approach taken by Kok et al. (2011). However, there could be open alternatives coexisting such as in the car example or in the gelato example. In these cases, a participant in the dialogue finds that there is a new alternative to be proposed when new circumstances highlight different evidence. One solution could be to construct all the alternatives that are feasible by all agents at a certain point in the dialogue, and then refine the set of options through an initial stage of problem formulation as suggested in Atkinson and Bench-Capon (2007). In complex situations, however, such as for long sequences of action, it may be unfeasible to construct all alternatives that are relevant for a situation. This approach should be designed to proceed with a restricted set of options, where the dialogue is then interleaved with additional phases where agents use new information discovered in the dialogue to expand the set of options gradually and construct alternative courses of action from modification of existing options.

\subsection{Dealing with the revision of an issue}


The introduction of a new alternative changes the status of deliberation and this must be represented in a system that models deliberative dialogue. In order to do so, the system must allow for a revision of the knowledge of the agents, in particular of those who did not suggest the alternative. In light of our examples, we argue here that in the dialogue, during the revision of the issue phase, referred to as revision in the MHP model, the revision of the issue itself should be made more explicit, to track the different sets of options available for the agents. In the deliberation system of Kok et al. (2011), a dialectical tree for an option consists of a graph of arguments that originates from the option itself (the root of the tree). The alternatives that an agent may propose during dialogue are set at the initial stage and arguments are exchanged during the dialogue constructing a tree of arguments for each option. Hence, the tracking of options available is done by observing the origins of the dialectical trees where the options reside. Such an option can be evaluated as acceptable or not according to the arguments in the dialectical tree and the final choice is made observing preferences among acceptable options. As previously introduced, in the latest version of the Carneades Argumentation System, not only is it possible to track different issues during the dialogue, but the system is now able to revise the issue as the dialogue proceeds. Such a system may provide useful insights on how to deal with the revision of an issue in agent deliberation.

The revision of the issue itself may be subject of deliberation, for example, we may ask whether it is worth considering an alternative, or whether there is the need to reduce the number of alternatives. The option tracking will allow for agents to be able to shift the dialogue to a meta-level phase of deliberation about issue revision. In this phase, agents may also decide whether the dialogue is terminated or should terminate. In the computational models of argumentation-based deliberation previously discussed, a new alternative is proposed via a speech act propose(action). However, a new speech act that specifically indicates the revision of the issue introducing or removing a new alternative, or start a new subissue may be more helpful to represent the transition. Such a speech act could also be considered as one of a kind, which can be instantiated through different variations such as introduce a new alternative, remove an alternative, switch to a sub-issue, or revert back to the primary issue. This is especially important in the analysis of a deliberative dialogue. Walton, Toniolo and Norman (2016), for example, express a number of criteria for evaluating whether at the closure stage of deliberation the dialogue was successful. One of those criteria states that the success of a dialogue depends on the accuracy and completeness of the information regarding the circumstances of the case. The introduction of a new revise speech act which explicitly shows a revision, might help better identify how new information has influenced the new proposal. In addition, we may be able to draw further conclusion on the quality of the proposal and the deliberation reflecting on whether it was reasonable to revise the issue at that specific point in dialogue, whether the new proposal is based on evidence and the new proposal is relevant within the current deliberation.

The notion of relevance also needs some reconfiguration. There needs to be a notion of relevance of the alternatives proposed according to what an agent thinks it is possible to achieve based on the information available. We must also consider the relevance of the moves in the dialogue. Within existing frameworks, the latter is formally approached by Kok et al. (2011) with a notion of relevance of an attacking move defined as one that changes the acceptability of the proposal. However, as we have shown through our examples, when new information is exchanged, for example via a disclose speech act, the acceptability of the existing proposals may not necessarily change but rather new information initiates a phase of revision of the issue. The move of a revise speech act in turn may not always lead to a change on the acceptability of the existing proposals, hence a more flexible notion of relevance 
should be considered within a framework that allows for supporting arguments or explanations to be introduced during the dialogue.

\section{Conclusions}

The examples studied in this paper have shown that there is the need to recognize a fundamental distinction between four types of deliberation as indicated in Table 1. These different types share the characteristics highlighted by McBurney et al. (2007) of collaboration among participants in deciding what course of action to take in a given set of circumstances, with no initial commitment to specific solutions, but rather to a goal that has to be achieved. The typology that we propose through our analysis indicates that these types have additional distinctive features regarding how the issue is posed and revised, the objective of engagement, the introduction of new information and exploration of options.

\begin{tabular}{|l|l|l|l|l|}
\hline & $\begin{array}{l}\text { Dilemma-like } \\
\text { Deliberation }\end{array}$ & $\begin{array}{l}\text { Static Decision } \\
\text { Making } \\
\text { Deliberation }\end{array}$ & $\begin{array}{l}\text { Dynamic Decision } \\
\text { Making } \\
\text { Deliberation }\end{array}$ & $\begin{array}{l}\text { Problem Solving } \\
\text { Deliberation }\end{array}$ \\
\hline Initial Issue & $\begin{array}{l}\text { Should we do this } \\
\text { or not? }\end{array}$ & $\begin{array}{l}\text { How do we do } \\
\text { this? }\end{array}$ & $\begin{array}{l}\text { Decide Which } \\
\text { Action to Take }\end{array}$ & $\begin{array}{l}\text { Decide How to } \\
\text { Solve a Problem }\end{array}$ \\
\hline Issue Revision & No & No & Yes & Yes \\
\hline $\begin{array}{l}\text { Objective of } \\
\text { Engagement in a } \\
\text { Deliberation }\end{array}$ & $\begin{array}{l}\text { Decide Whether } \\
\text { to Do One Action }\end{array}$ & $\begin{array}{l}\text { Decide Which } \\
\text { Action to Take }\end{array}$ & $\begin{array}{l}\text { Decide Which } \\
\text { Action to Take }\end{array}$ & $\begin{array}{l}\text { Decide How to } \\
\text { Best Solve a } \\
\text { Problem }\end{array}$ \\
\hline New Information & No & No & Yes & Yes \\
\hline $\begin{array}{l}\text { Effect of New } \\
\text { Information }\end{array}$ & N/A & $\begin{array}{l}\text { Do not change } \\
\text { number of } \\
\text { Options }\end{array}$ & $\begin{array}{l}\text { Add / Remove } \\
\text { Options }\end{array}$ & $\begin{array}{l}\text { Add / Remove } \\
\text { Options }\end{array}$ \\
\hline $\begin{array}{l}\text { Exploration of } \\
\text { Options }\end{array}$ & $\begin{array}{l}\text { All Analyzed at } \\
\text { Once with Pro- } \\
\text { cons }\end{array}$ & $\begin{array}{l}\text { Add / Remove } \\
\text { Options }\end{array}$ & $\begin{array}{l}\text { All Analyzed at } \\
\text { Once with Pro- } \\
\text { cons, but When } \\
\text { New Options Are } \\
\text { Added, New Pro- } \\
\text { cons May be } \\
\text { Advanced }\end{array}$ & $\begin{array}{l}\text { Dynamic Process } \\
\text { Interleaving } \\
\text { Analysis of } \\
\text { Pro/cons for } \\
\text { Options with } \\
\text { Advancement of } \\
\text { New Options }\end{array}$ \\
\hline
\end{tabular}

Table 1: Four Types of Deliberation

Most obvious is the kind of deliberation with fixed multiple choices often associated with the dilemma, where there is a fixed choice between mutually exclusive options and a more flexible problem solving type of deliberation concerning the evolution of changing means of solving a problem as new information comes in. The practical reasoning argumentation scheme is especially important in the problem solving type of deliberation both for posing a problem and for solving it by group deliberation.

Our six examples serve to showcase instances of decision making, such as the car buying example, where in its two options version it represents a common example proposed for what we refer here as static decision making deliberation. However, taking a broader perspective, this can be classified as the dynamic type, if more options are added or removed. Two of our examples showcase traits of problem solving only: the printer for an individual dialogue and the disaster as a collaborative dialogue, both aiming at identifying a course of action to solve a problem. The dialogue in the bin example and the COSTAR example, in addition to problem solving, interleave decision making. Finally, the education example 
represents an instance of a dialogue that although focussing on what to do in a specific situation presenting arguments that might fit with a practical reasoning scheme, the dialogue does not fit the deliberation model as the author is not in a position to make the decision.

We have shown that the dynamic decision making and problem solving deliberation types share characteristics of problem solving and decision theory in Artificial Intelligence and other disciplines. Our theory of deliberation dialogue is not meant to replace the classical method of problem solving found in the textbooks. We hope that its effect will be to enhance that method by providing a new tool that can be applied to instances where the multiagent communication between agents or among groups of agents is an important part of the problem solving procedure.

Our classification system is merely a tentative way of moving forward to try to make sense of what has now become a confused area with uncertainty about how argumentation can be useful for decision making and problem solving. We think we have provided a way forward that can lead to further fruitful discussion. Both decision theory and problem solving are huge fields in computing, operations research and many other areas. We can hardly make dogmatic pronouncements that the experts in these areas might be inclined to accept. But our attempt in this paper is to raise questions about the borderlines between decision making, problem solving, and other widely accepted technologies in relation to formal dialogue models of deliberation. Otherwise, surely these formal dialogue systems cannot move forward in so far as they claim to model real examples of argumentation that look like they should be exclusively treated by these well-established technologies.

The examples that we presented in this paper suggested other characteristics as well that have not previously been investigated. In particular, we proposed that the problem solving type of deliberation is characterised by the revision of the issue made necessary by the agents' increased knowledge of new circumstances. MCDM in particular needs to be extended to allow agents to formulate new alternatives as the dialogue proceeds on the basis of the information received and to argue about them based on factual evidence that is also open to dispute. MCDM would also need to allow for the utility function(s) to be revised as criteria change their importance during dialogue. The revision of the issue leads to further considerations on closing criteria and relevance of dialogue moves, hence it should be made more explicit via a new speech act, or many speech acts indicating various revision options, for example, in order to better analyse the outcome of deliberation. In conclusion, we showed that a more complete framework for deliberation is required to accommodate the features of realistic deliberation revealed by our examples.

\section{Acknowledgements}

Thanks are due to the Social Sciences and Humanities Research Council of Canada for Insight Grant 435-2012-0104: The Carneades Argumentation System. Part of this work was supported by the Scottish Informatics and Computer Science Alliance.

\section{References}

Aakhus, Mark, and Sally Jackson. 2005. Technology, interaction, and design. In Handbook of Language and Social Interaction, ed. Kristine L. Fitch and Robert E. Sanders. Lawrence Erlbaum Associates.

Anand, Paul. 1993. Foundations of Rational Choice Under Risk. Oxford University Press. 
Atkinson, Katie. 2005. What Should We Do? Computational Representation of Persuasive Argument in Practical Reasoning. PhD Thesis. University of Liverpool. https://cgi.csc.liv.ac.uk/ katie/ThesisFinal.pdf

Atkinson, Katie, Trevor Bench-Capon, and Peter McBurney. 2006. Computational representation of practical argument. Synthese 152: 157-206. doi:10.1007/s11229-005$3488-2$.

Atkinson, Katie, and Trevor Bench-Capon. 2007. Practical reasoning as presumptive argumentation using action based alternating transition systems. Artificial Intelligence 171(10-15): 855-874.

Atkinson, Katie, Trevor Bench-Capon, and Douglas Walton. 2013. Distinctive features of persuasion and deliberation dialogues. Argument and Computation 2: 105-127. doi:10.1080/19462166.2012.708670.

Bench-Capon, Trevor. 2003. Persuasion in practical argument using value-based argumentation frameworks. Journal of Logic and Computation 13: 429-448.

Black, Elisabeth, and Anthony Hunter. 2009. An inquiry dialogue system. Autonomous Agents and Multi-Agent Systems 19: 173-209.

Black, Elizabeth, and Katie Atkinson. 2009. Dialogues that account for different perspectives in collaborative argumentation. In Proceedings of the Eighth International Conference on Autonomous Agents and Multiagent Systems, 867-874.

Black, Elizabeth, and Katie Atkinson. 2011. Agreeing what to do. In Argumentation in MultiAgent Systems, ed. P. McBurney, I. Rahwan, and S. Parsons, 6614:12-30. Lecture Notes in Computer Science. Springer Berlin Heidelberg.

Bransford, John D, and Barry S Stein. 1993. The IDEAL Problem Solver: A Guide for Improving Thinking, Learning, and Creativity (Second Edition). NY: WH Freeman and Company.

Brewka, Gerhard, and Thomas F. Gordon. 1994. How to buy a porsche: An approach to defeasible decision making. In Working Notes of the AAAI-94 Workshop on Computational Dialectics. Seattle, Washington, 28-38.

Churchman, C West. 1971. The Design of Inquiring Systems: Basic Concepts of Systems and Organization. New York: Basic Books.

Erol, Kutluhan, James Hendler, and Dana S. Nau. 1995. HTN planning: Complexity and expressivity. In Proceedings of the Twelfth National Conference on Artificial Intelligence, 1123-1128.

Gordon, Thomas F. 2010. An overview of the Carneades argumentation support system. In Dialectics, Dialogue and Argumentation., ed. C. Reed and C. W. Tindale. London: College Publications. 
Gordon, Thomas F. 2015. Still craving a Porsche. In Advances in Knowledge Representation, Logic Programming, and Abstract Argumentation: Essays Dedicated to Gerhard Brewka on the Occasion of his 60th Birthday, 356-359. Vol. 9060: Springer.

Gordon, Thomas F. 2018. Defining Argument Weighing Functions, IFColog Journal of Logics and their Applications, 5(3), 747-773.

Gordon, Thomas F, and Douglas Walton. 2016. Formalizing balancing arguments. In Computational Models of Argument, ed. P. Baroni, T. F. Gordon, and T. Scheffler, 327338. Frontiers in Artificial Intelligence and Applications. IOS Press.

Gordon, Thomas F., Horst Friedrich, and Douglas Walton. 2018. Representing argumentation schemes with constraint handling rules (CHR). Argument and Computation 9(2): 91119. doi:10.3233/AAC-180039.

Grasso, Floriana. 2015. A case for motivational argumentation. In Proceedings of the 1st European Conference on Argumentation: Argumentation and Reasoned Action, vol 2 http://ecargument.org/wp-content/uploads/2015/05/ECA_Lisbon_2015_BOA.pdf

Hamblin, Charles, 1970. Fallacies, London: Methuen.

Hohmann, Hanns. 1989. The dynamics of stasis: Classical rhetorical theory and modern legal argumentation. American Journal of Jurisprudence 34: 171-197.

Hulstijn, Joris, and Leendert van der Torre. 2004. Combining goal generation and planning in an argumentation framework. In Proceedings of the Non-monotonic Reasoning Workshop on Argument, Dialogue and Decision, 212-218.

Jacobs, Scott. 1986. How to make an argument from examples in discourse analysis. In D. G. Ellis and W. A. Donohue (eds), Contemporary Issues in Language and Discourse Processes, Hillsdale, N.J.: Erlbaum, 149-168.

Klein, Mark. 2017. Towards Crowd-Scale Deliberation. SSRN Electronic Journal. https://ssrn.com/abstract=2987624.

Klein, Mark, and Luca Iandoli. 2008. Supporting Collaborative Deliberation Using a LargeScale Argumentation System: The MIT Collaboratorium. SSRN Electronic Journal MIT Sloan, Research Paper No. 4691-08. https://ssrn.com/abstract=1099082 or http://dx.doi.org/10.2139/ssrn.1099082

Kok, Eric M., John-Jules Ch. Meyer, Henry Prakken, and Gerard A. W. Vreeswijk. 2011. A Formal Argumentation Framework for Deliberation Dialogues. In Argumentation in Multi-Agent Systems, ed. P. McBurney, I. Rahwan, and S. Parsons, 6614: 31-48. Lecture Notes in Computer Science. Springer Berlin Heidelberg.

Leon, Marianthi, and Alice Toniolo. 2015. Analysis of collaborative design and decision making through argumentation applied for pre-BIM stages. Building Information Modelling (BIM) in Design, Construction and Operation, 149: 217-228. doi:10.2495/BIM150191 
Mason, Richard O. 1969. A Dialectical Approach to Strategic Planning. Management Science 15: B-403-B-414. doi:10.1287/mnsc.15.8.B403.

McBurney, Peter, David Hitchcock, and Simon Parsons. 2007. The eightfold way of deliberation dialogue. International Journal of Intelligent Systems 22(1): 95-132.

McBurney, Peter, and Simon Parsons. 2002. Games that agents play: A formal framework for dialogues between autonomous agents. Journal of Logic, Language and Information 11(3): 315-334.

Medellin-Gasque, Rolando, Katie Atkinson, Peter McBurney, and Trevor Bench-Capon. 2011. Arguments over co-operative plans. In Theory and Applications of Formal Argumentation, ed. S. Modgil, N. Oren, F. Toni, 7132: 50-66. Lecture Notes in Computer Science. Springer Berlin Heidelberg.

Mercier, Hugo, and Dan Sperber. 2011. Why do humans reason? Arguments for an argumentative theory. Behavioral and brain sciences 34(2). Cambridge Univ. Press: 57 74.

Mitroff, Ian I, James R Emshoff, and Ralph H Kilmann. 1979. Assumptional Analysis: A Methodology for Strategic Problem Solving. Management Science 25(6): 583-593. doi: $10.1287 / \mathrm{mnsc} .25 .6 .583$.

Mitroff, Ian, and Ralph Kilmann. 1978. On integrating behavioral and philosophical systems: Towards a unified theory of problem solving. Annual Series in Sociology 1: 207-236.

Munier, Nolberto, Eloy Hontoria, and Fernando Jiménez-Sáez. 2019. Strategic Approach in Multi-Criteria Decision Making: A Practical Guide for Complex Scenarios. International Series in Operations Research \& Management Science. Springer. doi:10.1007/978-3-030-02726-1.

Ouerdane, Wassila, Yannis Dimopoulos, Konstantinos Liapis, and Pavlos Mora, 2011. Towards automating decision aiding through argumentation. Journal of Multi-Criteria Decision Analysis, 18(5-6):289-309.

Poole, David L, and Alan K Mackworth. 2010. Artificial intelligence: foundations of computational agents. Cambridge University Press.

Prakken, Henry. 2005. Coherence and flexibility in dialogue games for argumentation. Journal of Logic and Computation 15(6): 1009-1040.

Prakken, Henry, and Giovanni Sartor. 2009. A logical analysis of burdens of proof. In Legal evidence and proof: Statistics, stories, logic, ed. H. Kaptein, H. Prakken, and B. Verheij, 223-253. Ashgate Publishing Farnham.

Rahwan and Amgoud, 2007. An argumentation-based approach for practical reasoning. In Nicolas Maudet, Simon Parsons, and Iyad Rahwan, editors, ArgMAS, volume 4766 of Lecture Notes in Computer Science, pages 74- 90. 
Reed, Chris. 1998. Dialogue frames in agent communication. In Proceedings of the Third International Conference on Multi Agent Systems, 246-253. (ICMAS98), IEEE Press, Paris, pp246-253

Russell, Stuart, and Peter Norvig. 1995. Artificial Intelligence: A Modern Approach. Upper Saddle River New Jersey: Prentice-Hall.

Steele, Katie, and Orri H. Stefánsson. 2015. Decision Theory. The Stanford Encyclopedia of Philosophy (Winter 2015 Edition), ed. Edward N. Zalta. https://plato.stanford.edu/archives/win2016/entries/decision-theory/

Stumpf, Simone C., and Janet T McDonnell. 2002. Talking about team framing: using argumentation to analyse and support experiential learning in early design episodes. Design Studies 23(1): 5-23. doi: 10.1016/S0142-694X(01)00020-5

Tang, Yuqing, Timothy J. Norman, and Simon Parsons. 2009. A Model for Integrating Dialogue and the Execution of Joint Plans. In Proceedings of the Eighth International Conference of Autonomous Agents and Multiagent Systems, 883-890.

Toni, Francesca. 2008. Assumption-Based Argumentation for Epistemic and Practical Reasoning. In Computable Models of the Law, ed. P. Casanovas, G. Sartor, N. Casellas, and R. Rubino, 4884: 185-202. Lecture Notes in Computer Science. Springer Berlin Heidelberg.

Toniolo, Alice. 2013. Models of argument for deliberative dialogue in complex domains. University of Aberdeen. PhD thesis, Department of Computing Science, University of Aberdeen, UK.

Toniolo, Alice, Timothy J. Norman, and Katia Sycara. 2012. An empirical study of argumentation schemes for deliberative dialogue. In Proceedings of the Twentieth European Conference on Artificial Intelligence, 242: 756-761.

Van der Weide, Tom, 2011. Arguing to Motivate Decisions. PhD Thesis. Dutch Research School for Information and Knowledge Systems: https://dspace.library.uu.nl/handle/1874/210788

Van Eemeren, Frans H. 2017. Argumentation Theory and Argumentative Practices: A Vital but Complex Relationship, Informal Logic, 37(4), 322-350.

Van Eemeren, Frans H., Rob Grootendorst, Sally Jackson, and Scott Jacobs. 1993. Reconstructing Argumentative Discourse, Tuscaloosa, University of Alabama Press.

Walton, Douglas. 2006. Fundamentals of Critical Argumentation. Cambridge: Cambridge University Press.

Walton, Douglas. 2015. Goal-based Reasoning for Argumentation. Cambridge: Cambridge University Press.

Walton, Douglas N. 1991. Critical faults and fallacies of questioning. Journal of Pragmatics 15(4): 337-366. doi:10.1016/0378-2166(91)90035-V. 
Walton, Douglas and Krabbe, Erik C. W. 1995. Commitment in Dialogue. Albany: SUNY Press.

Walton, Douglas, and Fabrizio Macagno. 2016. Profiles of dialogue for relevance. Informal Logic 36(4), 2016, 523-562. doi:10.22329/il.v36i4.4586.

Walton, Douglas, and Alice Toniolo. 2016. Deliberation, Practical Reasoning and Problem Solving. In Proceedings of the Eleventh Conference of the Ontario Society for the Study of Argumentation ed. P. Bondy and L. Benacquista, Windsor, Ontario, 2016. http://www.dougwalton.ca/papers\%20in\%20pdf/16DelibPR.pdf

Walton, Douglas, Alice Toniolo, and Timothy J. Norman. 2014. Missing phases of deliberation dialogue for real applications. In Proceedings of the Eleventh International Workshop on Argumentation in Multi-Agent Systems.

Walton, Douglas, Alice Toniolo, and Timothy J. Norman. 2016. Towards a richer model of deliberation dialogue: Closure problem and change of circumstances. Argument and Computation 7: 155-173. doi: 10.3233/AAC-160009

Yao, Shengji. 2007. Experimental approaches for understanding conceptual design activities. Department of Mechanical and Industrial Engineering, Concordia University, Canada. 